Acta Crystallographica Section E

\section{Structure Reports}

Online

ISSN 1600-5368

\section{Erythromycin A dimethyl sulfoxide disolvate 1.43-hydrate}

\author{
Jürgen Brüning, ${ }^{a}$ Tanja K. Trepte, ${ }^{a}$ Jan W. Bats ${ }^{b}$ and \\ Martin U. Schmidt ${ }^{\mathrm{a} *}$
}

anstitute of Inorganic and Analytical Chemistry, University of Frankfurt, Max-vonLaue-Strasse 7, 60438 Frankfurt, Germany, and ${ }^{\mathbf{b}}$ Institute of Organic Chemistry and Chemical Biology, University of Frankfurt, Max-von-Laue-Strasse 7, 60438 Frankfurt, Germany

Correspondence e-mail: m.schmidt@chemie.uni-frankfurt.de

Received 27 January 2012; accepted 6 February 2012

Key indicators: single-crystal X-ray study; $T=296 \mathrm{~K}$; mean $\sigma(\mathrm{C}-\mathrm{C})=0.005 \AA$; $\mathrm{H}$ atom completeness $99 \%$; disorder in solvent or counterion; $R$ factor $=0.062$; $w R$ factor $=0.142 ;$ data-to-parameter ratio $=18.2$.

The title compound, $\mathrm{C}_{37} \mathrm{H}_{67} \mathrm{NO}_{13} \cdot 2 \mathrm{C}_{2} \mathrm{H}_{6} \mathrm{OS} \cdot 1.43 \mathrm{H}_{2} \mathrm{O}$, is a macrolide antibiotic with better solubility and better dermal penetration abilities than erythromycin A itself. The asymmetric unit of this form contains one erythromycin A molecule, two dimethyl sulfoxide (DMSO) solvent molecules, a fully occupied water molecule and a partially occupied water molecule with an occupancy factor of 0.432 (11). The 14membered ring of the erythronolide fragment has a conformation which differs considerably from that in erythromycin A dihydrate [Stephenson, Stowell, Toma, Pfeiffer \& Byrn (1997). J. Pharm. Sci. 86, 1239-1244]. One of the two DMSO molecules is disordered over two orientations; the orientation depends on the presence or absence of the second, partially occupied, water molecule. In the crystal, erythromycin molecules are connected by $\mathrm{O}-\mathrm{H} \cdots \mathrm{O}$ hydrogen bonds involving the hydroxy groups and the fully occupied water molecule to form layers parallel to (010). These layers are connected along the $b$-axis direction only by a possible hydrogen-bonding contact involving the partially occupied water molecule.

\section{Related literature}

For a description of the title compound, see: Schmidt et al. (2011). For general background, see: Woodward et al. (1981). For crystallization experiments, see: Mirza et al. (2003). For related structures, see: Stephenson et al. (1997); Henry \& Zhang (2007); Tian et al. (2009). For refinement details, see: Flack (1983); Spek (2009).

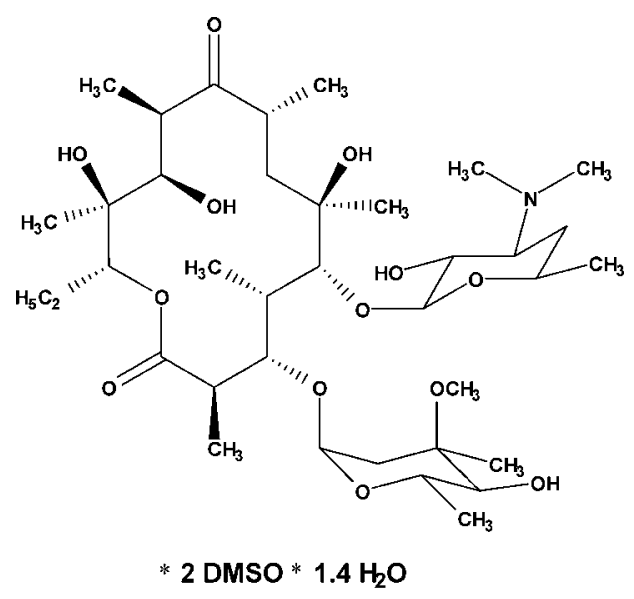

\section{Experimental}

Crystal data

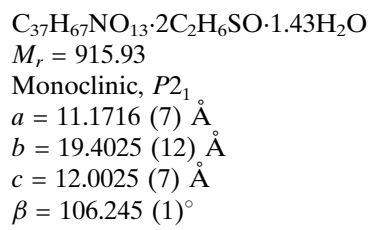

\section{Data collection}

Siemens SMART 1K CCD diffractometer

Absorption correction: multi-scan (SADABS; Sheldrick, 2000)

$$
T_{\min }=0.870, T_{\max }=0.947
$$

Refinement

$R\left[F^{2}>2 \sigma\left(F^{2}\right)\right]=0.062$

$w R\left(F^{2}\right)=0.142$

$S=1.04$

10520 reflections

579 parameters

23 restraints

$$
\begin{aligned}
& V=2497.8(3) \AA^{3} \\
& Z=2 \\
& \text { Mo } K \alpha \text { radiation } \\
& \mu=0.17 \mathrm{~mm}^{-1} \\
& T=296 \mathrm{~K} \\
& 0.55 \times 0.42 \times 0.32 \mathrm{~mm}
\end{aligned}
$$

27453 measured reflections

\begin{tabular}{|c|c|c|c|c|}
\hline$D-\mathrm{H} \cdots A$ & $D-\mathrm{H}$ & $\mathrm{H} \cdots A$ & $D \cdots A$ & $D-\mathrm{H} \cdots A$ \\
\hline $\mathrm{O} 6-\mathrm{H} 6 A \cdots \mathrm{O} 7 W^{\mathrm{i}}$ & 0.82 & 2.00 & $2.810(4)$ & 170 \\
\hline $\mathrm{O} 7 W-\mathrm{H} 7 C \cdots \mathrm{O} 26^{\mathrm{ii}}$ & $0.85(1)$ & $2.07(2)$ & $2.888(4)$ & $163(4)$ \\
\hline $\mathrm{O} 7 W-\mathrm{H} 7 D \cdots \mathrm{N} 33$ & $0.84(1)$ & $2.04(2)$ & $2.863(4)$ & $166(5)$ \\
\hline $\mathrm{O} 11-\mathrm{H} 11 B \cdots \mathrm{O} 12$ & 0.82 & 2.07 & $2.575(4)$ & 120 \\
\hline $\mathrm{O} 12-\mathrm{H} 12 A \cdots \mathrm{O} 15$ & 0.82 & 1.85 & $2.602(8)$ & 152 \\
\hline $\mathrm{O} 12-\mathrm{H} 12 A \cdots \mathrm{O}^{\prime} 5^{\prime}$ & 0.82 & 2.04 & $2.667(7)$ & 134 \\
\hline $\mathrm{O} 25-\mathrm{H} 25 B \cdots \mathrm{O} 11^{\mathrm{iii}}$ & 0.82 & 2.04 & $2.838(4)$ & 166 \\
\hline $\mathrm{O} 34-\mathrm{H} 34 B \cdots \mathrm{O} 14$ & 0.82 & 1.94 & $2.747(4)$ & 170 \\
\hline
\end{tabular}
10520 independent reflections 7109 reflections with $I>2 \sigma(I)$ $R_{\text {int }}=0.037$

\title{
(23 restraints
}

Table 1

Hydrogen-bond geometry $\left(\AA,^{\circ}\right)$.

Symmetry codes: (i) $x+1, y, z$; (ii) $x-1, y, z$; (iii) $x, y, z+1$.

Data collection: SMART (Siemens, 1995); cell refinement: SAINT (Siemens, 1995); data reduction: $S A I N T$; program(s) used to solve structure: SHELXS97 (Sheldrick, 2008); program(s) used to refine structure: SHELXL97 (Sheldrick, 2008); molecular graphics: SHELXTL (Sheldrick, 2008); software used to prepare material for publication: SHELXL97. 
The authors gratefully thank Edith Alig for the X-ray powder diffraction experiments.

Supplementary data and figures for this paper are available from the IUCr electronic archives (Reference: SJ5188).

\section{References}

Flack, H. D. (1983). Acta Cryst. A39, 876-881.

Henry, R. \& Zhang, G. G. Z. (2007). J. Pharm. Sci. 96, 1251-1257.

Mirza, S., Miroshnyk, I., Heinämäki, J., Christiansen, L., Karjalainen, M. \& Yliruusi, J. (2003). AAPS PharmSci. 5(2), 39-47.
Schmidt, M. U., Brüning, J., Trepte, T. K. \& Bats, J. W. (2011). German Patent Appl. 102011117874.4.

Sheldrick, G. M. (2000). SADABS. University of Göttingen, Germany.

Sheldrick, G. M. (2008). Acta Cryst. A64, 112-122.

Siemens (1995). SMART and SAINT. Siemens Analytical X-ray Instruments Inc., Madison, Wisconsin, USA.

Spek, A. L. (2009). Acta Cryst. D65, 148-155.

Stephenson, G. A., Stowell, J. G., Toma, P. H., Pfeiffer, R. R. \& Byrn, S. R. (1997). J. Pharm. Sci. 86, 1239-1244.

Tian, J., Thallapally, P. K., Dalgarno, S. J. \& Atwood, J. L. (2009). J. Am. Chem. Soc. 131, 13216-13217.

Woodward, R. B., Logusch, E., Nambiar, K. P., Sakan, K., Ward, D. E., AuYeung, B. W., Balaram, P., Browne, L. J., Card, P. J. \& Chen, C. H. (1981). J. Am. Chem. Soc. 103, 3210-3213. 


\section{supporting information}

Acta Cryst. (2012). E68, o700-o701 [doi:10.1107/S1600536812005223]

\section{Erythromycin A dimethyl sulfoxide disolvate 1.43-hydrate}

\section{Jürgen Brüning, Tanja K. Trepte, Jan W. Bats and Martin U. Schmidt}

\section{S1. Comment}

Erythromycin is a macrolide antibiotic with a wide antimicrobial spectrum (Woodward et al., 1981; Schmidt et al., 2011). The dimethyl sulfoxide disolvate 1.4-hydrate of erythromycin A reported here may have potential as an active pharmaceutical ingredient (API), because it shows enhanced solubility and better dermal penetration abilities than erythromycin A itself (Schmidt et al., 2011). The title compound can be used as an antibiotic, e.g. for defence against germs such as bacillus anthracis, streptococcaceae, bordetella, legionellaceae, and chlamydiaceae. It could also have a role in the treatment of infections, e.g. in the ear, nose, and throat regions, especially in the middle ear and the paranasal sinuses. It could be used to treat infections in the deep respiratory passages against e.g. bronchitis, pneumonia, pertussis, and infection of the conjunctiva. Other uses include the treatment of erysipelas, diphtheria, severe forms of acne vulgaris, inflammation of the skin and urethra, and dysfunction of motility and excretion. It could treat inflammation of the gastro and intestinal tract as well as inflammation of the pharynx, e.g. pharyngitis, tonsillitis, or scarlatina, syphilis and actinomycosis. It is a potential prophylactic against rheumatic fever after infection, especially in case of penicillin allergy. The compound may also be applied to comparable diseases in veterinary medicine as well. The crystalline forms of a number of other solvates of erythromycin A have been characterized by Stephenson et al. (1997), Mirza et al. (2003) and Henry \& Zhang (2007).

The asymmetric unit of the dimethyl sulfoxide disolvate 1.4-hydrate of erythromycin A, (I, Fig. 1), $\mathrm{C}_{37} \mathrm{H}_{67} \mathrm{NO}_{13} .2\left(\mathrm{C}_{2} \mathrm{H}_{6} \mathrm{OS}\right) .1 .4\left(\mathrm{H}_{2} \mathrm{O}\right)$, at $296 \mathrm{~K}$ contains one erythromycin A molecule, two DMSO solvate molecules, a fully occupied water molecule and a partially occupied water molecule with an occupancy factor of 0.432 (11). The 14membered ring has a conformation which differs considerably from that in the crystal structure of erythromycin A dihydrate (Stephenson et al., 1997). For the two structures, the torsion angles in the fragment C10-C11-C12-C13-O13$\mathrm{C} 1-\mathrm{C} 2-\mathrm{C} 3-\mathrm{C} 4-\mathrm{C} 5-\mathrm{C} 6$ are rather similar, but torsion angles in the fragment $\mathrm{C} 6-\mathrm{C} 7-\mathrm{C} 8-\mathrm{C} 9-\mathrm{C} 10$ are considerably different. The six-membered rings of the side-chains have the usual chair conformation. The partially occupied water

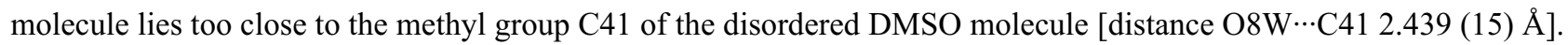
Thus, if the water molecule is present, the disordered DMSO molecule must have the orientation defined by positions S2', $\mathrm{C} 40^{\prime}, \mathrm{C} 41^{\prime}, \mathrm{O} 15^{\prime}$. A search for possible solvent accessible voids with program PLATON (Spek, 2009) reveals that no voids are present in the unit cell if the disordered DMSO molecule has the orientation defined by positions S2, O15, C40, C41, but that a void of $16 \AA^{3}$ at the position of atom O8W occurs when this DMSO molecule has the orientation defined by positions $\mathrm{S} 2^{\prime}, \mathrm{O}^{\prime} 5^{\prime}, \mathrm{C} 40^{\prime}, \mathrm{C} 41^{\prime}$. No $\mathrm{H}$ atoms were located at the partially occupied water molecule (O8W). However,

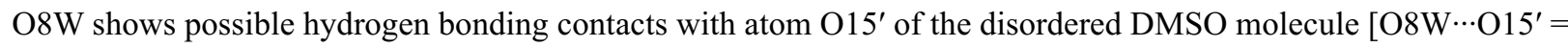

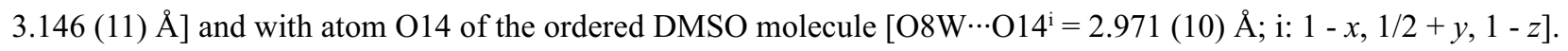
The $\mathrm{O} 15^{\prime}-\mathrm{O} 8 \mathrm{~W}-\mathrm{O} 14^{\mathrm{i}}$ angle of $139.8(4)^{\circ}$, however, appears too large for both hydrogen bonds to occur simultaneously. A partially occupied water molecule has also been observed in the crystal structure of the closely related compound clarithromycin (Tian et al., 2009). The fully occupied water molecule (O7W, H7C, H7D) accepts a hydrogen 


\section{supporting information}

bond from the hydroxy group O6- $-\mathrm{H} 6 \mathrm{~A}$ and acts as a donor for two hydrogen bonds to two symmetry-related erythromycin molecules (Table 1).

The erythromycin molecule shows one intramolecular $\mathrm{O}-\mathrm{H} \cdots \mathrm{O}$ hydrogen bond. The remaining hydroxy groups are involved in intermolecular hydrogen bonds. One of them links the erythromycin molecules along the $c$-axis direction. The other three hydroxy groups are directed towards the two DMSO solvate molecules and towards water molecule O7W. The latter water molecule links the erythromycin molecules along the $a$-axis direction. Thus, the structure contains hydrogen bonded layers parallel to the (010) plane. The only intermolecular contact between adjacent layers along the $b$ axis direction is the before mentioned possible $\mathrm{O} 8 \mathrm{~W} \cdots \mathrm{O} 14^{\mathrm{i}}$ hydrogen bond, involving the partially occupied water molecule.

A second crystal of the title compound was measured at $177 \mathrm{~K}$. The resulting crystal structure was similar to the structure determined at room temperature. The occupancy factor of the partially occupied water molecule refined to 0.400 (7). No phase transition was observed on cooling of the crystal from room temperature to $177 \mathrm{~K}$.

\section{S2. Experimental}

Erythromycin A was recrystallized from a dimethyl sulfoxide/water mixture. The crystal used for the data collection was sealed in a glass capillary tube with a drop of mother liquor.

The compound was also characterized by its X-ray powder pattern which shows the following characteristic reflections $\left(\mathrm{Cu} \mathrm{K} \alpha_{1}\right.$ radiation, $2 \theta$ in ${ }^{\circ}$, rel. intensities: ss = very strong, $\mathrm{s}=$ strong, $\mathrm{m}=$ medium, $\mathrm{mw}=$ medium-weak, $\mathrm{w}=$ weak $): 7.6$ (w), $9.1(\mathrm{ss}), 9.4(\mathrm{ss}), 9.5(\mathrm{~m}), 11.9(\mathrm{~m}), 12.3(\mathrm{~s}), 13.2(\mathrm{mw}), 13.5(\mathrm{mw}), 15.3(\mathrm{w}), 15.6(\mathrm{~s}), 15.9(\mathrm{mw}), 16.7(\mathrm{ss}), 17.1(\mathrm{w})$, $17.8(\mathrm{w}), 18.2(\mathrm{mw}), 18.5(\mathrm{w}), 18.7(\mathrm{w}), 18.9(\mathrm{mw}), 19.1(\mathrm{w}), 19.4(\mathrm{~m}), 19.7(\mathrm{~m}), 19.9(\mathrm{mw}), 20.5(\mathrm{~m}), 21.5(\mathrm{~m}), 22.1(\mathrm{w})$, $22.3(\mathrm{mw}), 22.7(\mathrm{mw}), 23.6(\mathrm{w}), 23.9(\mathrm{w}), 24.1(\mathrm{w}), 24.4(\mathrm{mw}), 24.8(\mathrm{w}), 26.1(\mathrm{w}), 26.2(\mathrm{w}), 26.6(\mathrm{w}), 26.9(\mathrm{w}), 27.2(\mathrm{w})$, 27.6(w), $28.1(\mathrm{w}), 28.4(\mathrm{w}), 28.9(\mathrm{w}), 29.6(\mathrm{w}), 30.0(\mathrm{w}), 30.1(\mathrm{w}), 31.3(\mathrm{w}), 31.6(\mathrm{w}), 32.1(\mathrm{w}), 33.7(\mathrm{w}), 34.3(\mathrm{w}), 36.2$ (w), 36.4(w), 37.6 (w), 37.8 (w), 38.9(w), 39.3 (w).

\section{S3. Refinement}

The $\mathrm{H}$ atoms were positioned geometrically and treated as riding with $\mathrm{C}_{\text {primary }}-\mathrm{H}=0.98 \AA, \mathrm{C}_{\text {secondary }}-\mathrm{H}=0.97 \AA, \mathrm{C}_{\text {methyl }}$ $-\mathrm{H}=0.96 \AA, \mathrm{O}-\mathrm{H}=0.82 \AA, U_{\text {iso }}(H)=1.2 U_{\text {eq }}\left(\mathrm{C}_{\text {non-methyl }}\right)$ and $U_{\text {iso }}(H)=1.5 U_{\text {eq }}\left(\mathrm{C}_{\text {methyl }}, O\right)$. The $\mathrm{H}$ atoms at water molecule $\mathrm{O} 7 \mathrm{~W}$ were taken from a difference Fourier synthesis and were refined, using an $\mathrm{O}-\mathrm{H}$ distance restraint of 0.84 (1) $\AA$. The $\mathrm{O}$ and $\mathrm{C}$ atoms of the disordered DMSO solvate molecule were refined as anisotropic split atoms, using 20 restraints to bond distances and atomic displacement parameters. The occupancy factors refined to 0.482 (4) for atoms O15, C40, $\mathrm{C} 41$ and to 0.518 (4) for atoms $\mathrm{O}^{\prime} 5^{\prime}, \mathrm{C} 40^{\prime}, \mathrm{C} 41^{\prime}$. The occupancy factor of the water molecule O8W refined to 0.432 (11). $\mathrm{H}$ atoms associated with the partially occupied water molecule $(\mathrm{O} 8 \mathrm{~W})$ could not be located in difference Fourier maps and were not assigned. 

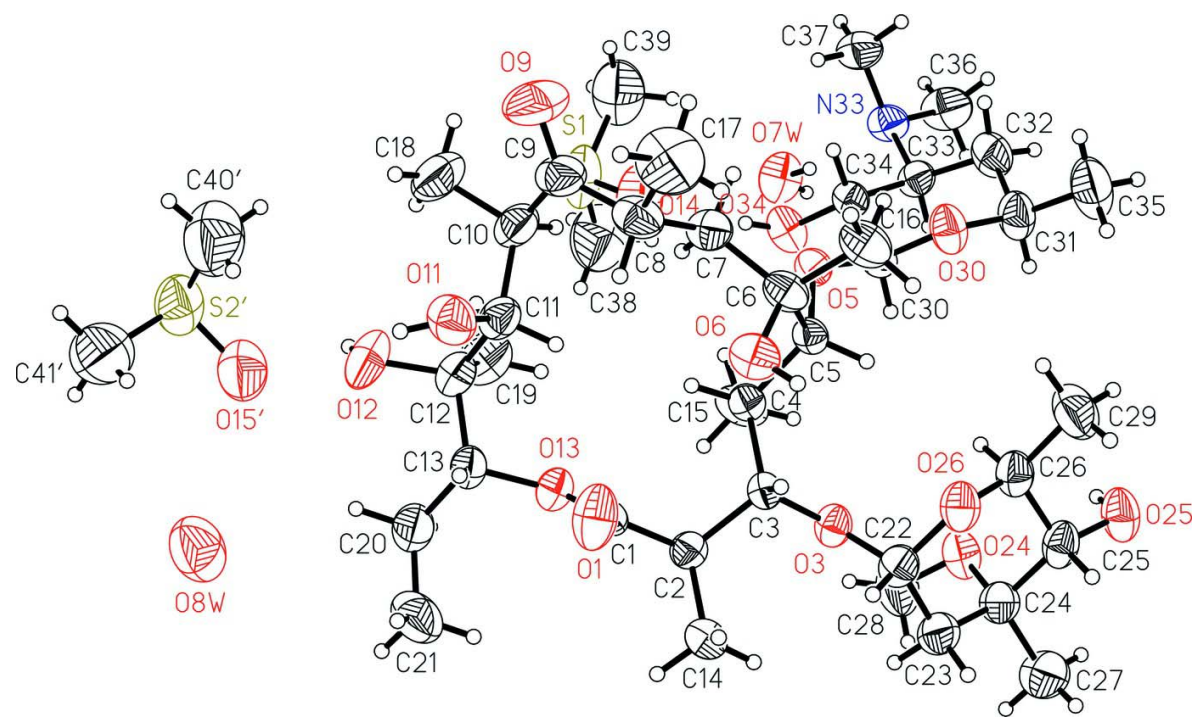

\section{Figure 1}

The asymmetric unit of (I) at $296 \mathrm{~K}$ with the numbering scheme of the atoms. Displacement ellipsoids are drawn at the $50 \%$ probability level and $\mathrm{H}$ atoms are shown as small spheres of arbitrary radii. Atoms belonging to the second orientation of the disordered DMSO solvate molecule have been omitted for clarity.

\section{Erythromycin A dimethyl sulfoxide disolvate 1.43-hydrate}

\section{Crystal data}

$\mathrm{C}_{37} \mathrm{H}_{67} \mathrm{NO}_{13} \cdot 2 \mathrm{C}_{2} \mathrm{H}_{6} \mathrm{SO} \cdot 1.43 \mathrm{H}_{2} \mathrm{O}$

$M_{r}=915.93$

Monoclinic, $P 2_{1}$

Hall symbol: $\mathrm{P} 2 \mathrm{yb}$

$a=11.1716(7) \AA$

$b=19.4025(12) \AA$

$c=12.0025(7) \AA$

$\beta=106.245(1)^{\circ}$

$V=2497.8(3) \AA^{3}$

$Z=2$

\section{Data collection}

\section{Siemens SMART 1K CCD}

diffractometer

Radiation source: normal-focus sealed tube

Graphite monochromator

$\omega$ scans

Absorption correction: multi-scan

(SADABS; Sheldrick, 2000)

$T_{\min }=0.870, T_{\max }=0.947$

\section{Refinement}

Refinement on $F^{2}$

Least-squares matrix: full

$R\left[F^{2}>2 \sigma\left(F^{2}\right)\right]=0.062$

$w R\left(F^{2}\right)=0.142$

$S=1.04$

10520 reflections
$F(000)=997$

$D_{\mathrm{x}}=1.218 \mathrm{Mg} \mathrm{m}^{-3}$

Mo $K \alpha$ radiation, $\lambda=0.71073 \AA$

Cell parameters from 6074 reflections

$\theta=3-24^{\circ}$

$\mu=0.17 \mathrm{~mm}^{-1}$

$T=296 \mathrm{~K}$

Block, colourless

$0.55 \times 0.42 \times 0.32 \mathrm{~mm}$

27453 measured reflections

10520 independent reflections

7109 reflections with $I>2 \sigma(I)$

$R_{\text {int }}=0.037$

$\theta_{\text {max }}=27.0^{\circ}, \theta_{\text {min }}=2.1^{\circ}$

$h=-14 \rightarrow 14$

$k=-24 \rightarrow 24$

$l=-15 \rightarrow 15$

579 parameters

23 restraints

Primary atom site location: structure-invariant direct methods

Secondary atom site location: difference Fourier map 
Hydrogen site location: inferred from neighbouring sites

$\mathrm{H}$ atoms treated by a mixture of independent and constrained refinement

$w=1 /\left[\sigma^{2}\left(F_{\mathrm{o}}^{2}\right)+(0.062 P)^{2}+0.61 P\right]$

where $P=\left(F_{\mathrm{o}}^{2}+2 F_{\mathrm{c}}^{2}\right) / 3$
$(\Delta / \sigma)_{\max }<0.001$

$\Delta \rho_{\max }=0.37{\mathrm{e} \AA^{-3}}^{-3}$

$\Delta \rho_{\min }=-0.34$ e $\AA^{-3}$

Absolute structure: Flack (1983), with 4965

Friedel pairs

Absolute structure parameter: 0.02 (9)

Special details

Geometry. All e.s.d.'s (except the e.s.d. in the dihedral angle between two 1.s. planes) are estimated using the full covariance matrix. The cell e.s.d.'s are taken into account individually in the estimation of e.s.d.'s in distances, angles and torsion angles; correlations between e.s.d.'s in cell parameters are only used when they are defined by crystal symmetry. An approximate (isotropic) treatment of cell e.s.d.'s is used for estimating e.s.d.'s involving 1.s. planes.

Refinement. Refinement of $F^{2}$ against ALL reflections. The weighted $R$-factor $w R$ and goodness of fit $S$ are based on $F^{2}$, conventional $R$-factors $R$ are based on $F$, with $F$ set to zero for negative $F^{2}$. The threshold expression of $F^{2}>\sigma\left(F^{2}\right)$ is used only for calculating $R$-factors $(\mathrm{gt})$ etc. and is not relevant to the choice of reflections for refinement. $R$-factors based on $F^{2}$ are statistically about twice as large as those based on $F$, and $R$-factors based on ALL data will be even larger.

Fractional atomic coordinates and isotropic or equivalent isotropic displacement parameters $\left(\AA^{2}\right)$

\begin{tabular}{|c|c|c|c|c|c|}
\hline & $x$ & $y$ & $z$ & $U_{\text {iso }} * / U_{\text {eq }}$ & Occ. $(<1)$ \\
\hline $\mathrm{O} 1$ & $0.9259(2)$ & $0.73051(18)$ & $0.7044(2)$ & $0.0678(8)$ & \\
\hline $\mathrm{O} 3$ & 0.89509 (19) & $0.71189(13)$ & $1.06566(17)$ & $0.0416(5)$ & \\
\hline O5 & 0.66199 (19) & $0.54287(13)$ & $0.93991(17)$ & $0.0379(5)$ & \\
\hline O6 & $0.9249(2)$ & $0.56635(14)$ & $0.8216(2)$ & $0.0498(6)$ & \\
\hline H6A & 0.9900 & 0.5650 & 0.8743 & $0.075^{*}$ & \\
\hline O7W & $0.1470(3)$ & $0.57984(19)$ & $1.0016(2)$ & $0.0695(8)$ & \\
\hline $\mathrm{H} 7 \mathrm{C}$ & $0.129(4)$ & $0.592(3)$ & $1.063(2)$ & $0.11(2)^{*}$ & \\
\hline H7D & 0.207 (3) & $0.552(2)$ & $1.022(3)$ & $0.094(18)^{*}$ & \\
\hline O8W & $0.5845(8)$ & $0.9276(5)$ & $0.2582(8)$ & $0.106(4)$ & $0.432(11)$ \\
\hline O9 & $0.5978(4)$ & $0.47211(19)$ & 0.4648 & $0.0971(11)$ & \\
\hline $\mathrm{O} 11$ & 0.7227 & $0.63909(15)$ & $0.4476(2)$ & $0.0601(7)$ & \\
\hline H11B & 0.6961 & 0.6645 & 0.3916 & $0.090^{*}$ & \\
\hline $\mathrm{O} 12$ & $0.5552(3)$ & $0.73381(19)$ & $0.3910(2)$ & $0.0767(9)$ & \\
\hline $\mathrm{H} 12 \mathrm{~A}$ & 0.4817 & 0.7454 & 0.3714 & $0.115^{*}$ & \\
\hline O13 & $0.7336(2)$ & $0.77075(14)$ & $0.68886(18)$ & $0.0441(5)$ & \\
\hline $\mathrm{O} 24$ & $0.8552(2)$ & $0.73134(15)$ & $1.3084(2)$ & $0.0556(6)$ & \\
\hline $\mathrm{O} 25$ & $0.9804(2)$ & $0.62995(16)$ & $1.4629(2)$ & $0.0600(7)$ & \\
\hline $\mathrm{H} 25 \mathrm{~B}$ & 0.9057 & 0.6380 & 1.4505 & $0.090 *$ & \\
\hline $\mathrm{O} 26$ & $1.0377(2)$ & $0.63220(14)$ & $1.17612(19)$ & $0.0493(6)$ & \\
\hline $\mathrm{O} 30$ & $0.7064(2)$ & $0.47251(14)$ & $1.0976(2)$ & $0.0511(6)$ & \\
\hline O34 & $0.4210(2)$ & $0.56850(14)$ & $0.9577(2)$ & $0.0517(6)$ & \\
\hline H34B & 0.4199 & 0.5717 & 0.8893 & $0.078^{*}$ & \\
\hline N33 & $0.3193(3)$ & $0.46849(16)$ & $1.0750(2)$ & $0.0466(7)$ & \\
\hline $\mathrm{C} 1$ & $0.8498(3)$ & $0.75202(17)$ & 0.7486 & $0.0389(7)$ & \\
\hline $\mathrm{C} 2$ & 0.8725 & $0.76395(17)$ & 0.8779 & $0.0389(7)$ & \\
\hline $\mathrm{H} 2 \mathrm{~A}$ & 0.8037 & 0.7917 & 0.8895 & $0.047 *$ & \\
\hline C3 & $0.8777(3)$ & $0.69572(17)$ & $0.9450(2)$ & $0.0335(7)$ & \\
\hline $\mathrm{H} 3 \mathrm{~A}$ & 0.9491 & 0.6686 & 0.9375 & $0.040^{*}$ & \\
\hline C4 & 0.7580 & $0.65215(17)$ & 0.9018 & $0.0363(7)$ & \\
\hline
\end{tabular}




\begin{tabular}{|c|c|c|c|c|}
\hline $\mathrm{H} 4 \mathrm{~A}$ & 0.7360 & 0.6524 & 0.8168 & $0.044^{*}$ \\
\hline $\mathrm{C} 5$ & $0.7790(3)$ & $0.57594(17)$ & $0.9404(3)$ & $0.0345(7)$ \\
\hline H5A & 0.8368 & 0.5736 & 1.0185 & $0.041 *$ \\
\hline C6 & $0.8284(3)$ & $0.52994(18)$ & $0.8564(3)$ & $0.0441(8)$ \\
\hline $\mathrm{C} 7$ & $0.7203(3)$ & $0.5174(2)$ & $0.7452(3)$ & $0.0469(8)$ \\
\hline H7A & 0.6788 & 0.4750 & 0.7556 & $0.056^{*}$ \\
\hline H7B & 0.6605 & 0.5545 & 0.7385 & $0.056^{*}$ \\
\hline C8 & $0.7540(4)$ & 0.51235 (19) & 0.6293 & $0.0533(9)$ \\
\hline H8A & 0.8079 & 0.5515 & 0.6250 & $0.064 *$ \\
\hline C9 & $0.6353(4)$ & $0.5193(2)$ & 0.5315 & $0.0599(11)$ \\
\hline $\mathrm{C} 10$ & $0.5615(3)$ & $0.5868(2)$ & $0.5174(3)$ & $0.0555(10)$ \\
\hline H10A & 0.5242 & 0.5897 & 0.5821 & $0.067^{*}$ \\
\hline C11 & $0.6505(3)$ & $0.6485(2)$ & $0.5281(3)$ & $0.0479(9)$ \\
\hline H11A & 0.7088 & 0.6463 & 0.6061 & $0.058^{*}$ \\
\hline C12 & $0.5943(3)$ & $0.7213(2)$ & $0.5137(3)$ & $0.0515(9)$ \\
\hline C13 & $0.7001(3)$ & $0.7736(2)$ & 0.5620 & $0.0478(8)$ \\
\hline H13A & 0.7728 & 0.7604 & 0.5363 & $0.057^{*}$ \\
\hline C14 & $0.9918(4)$ & $0.8051(2)$ & $0.9199(3)$ & $0.0569(10)$ \\
\hline $\mathrm{H} 14 \mathrm{~A}$ & 0.9844 & 0.8471 & 0.8762 & $0.085^{*}$ \\
\hline H14B & 1.0603 & 0.7785 & 0.9093 & $0.085 *$ \\
\hline $\mathrm{H} 14 \mathrm{C}$ & 1.0063 & 0.8158 & 1.0007 & $0.085^{*}$ \\
\hline C15 & $0.6484(3)$ & $0.6862(2)$ & $0.9362(4)$ & $0.0573(10)$ \\
\hline H15A & 0.6401 & 0.7332 & 0.9105 & $0.086^{*}$ \\
\hline H15B & 0.6642 & 0.6847 & 1.0190 & $0.086^{*}$ \\
\hline $\mathrm{H} 15 \mathrm{C}$ & 0.5727 & 0.6617 & 0.9003 & $0.086^{*}$ \\
\hline C16 & $0.8815(4)$ & $0.46350(19)$ & $0.9167(4)$ & $0.0577(10)$ \\
\hline H16A & 0.9136 & 0.4359 & 0.8653 & $0.087^{*}$ \\
\hline H16B & 0.8169 & 0.4385 & 0.9379 & $0.087^{*}$ \\
\hline $\mathrm{H} 16 \mathrm{C}$ & 0.9474 & 0.4740 & 0.9853 & $0.087 *$ \\
\hline C17 & $0.8250(5)$ & 0.4457 & $0.6187(5)$ & $0.0940(17)$ \\
\hline H17A & 0.8444 & 0.4454 & 0.5457 & $0.141 *$ \\
\hline H17B & 0.7739 & 0.4065 & 0.6229 & $0.141 *$ \\
\hline $\mathrm{H} 17 \mathrm{C}$ & 0.9008 & 0.4436 & 0.6808 & $0.141 *$ \\
\hline C18 & $0.4532(4)$ & 0.5846 & $0.4040(4)$ & $0.0864(15)$ \\
\hline H18A & 0.4033 & 0.5444 & 0.4040 & $0.130 *$ \\
\hline H18B & 0.4866 & 0.5831 & 0.3385 & $0.130^{*}$ \\
\hline $\mathrm{H} 18 \mathrm{C}$ & 0.4025 & 0.6251 & 0.3991 & $0.130 *$ \\
\hline C19 & $0.4898(4)$ & $0.7309(3)$ & $0.5700(4)$ & $0.0748(12)$ \\
\hline H19A & 0.4251 & 0.6979 & 0.5386 & $0.112 *$ \\
\hline H19B & 0.4567 & 0.7767 & 0.5547 & $0.112 *$ \\
\hline H19C & 0.5215 & 0.7242 & 0.6522 & $0.112 *$ \\
\hline $\mathrm{C} 20$ & $0.6678(4)$ & $0.8489(2)$ & 0.5289 & $0.0692(12)$ \\
\hline $\mathrm{H} 20 \mathrm{~A}$ & 0.6292 & 0.8517 & 0.4459 & $0.083^{*}$ \\
\hline $\mathrm{H} 20 \mathrm{~B}$ & 0.6076 & 0.8649 & 0.5677 & $0.083^{*}$ \\
\hline $\mathrm{C} 21$ & $0.7804(5)$ & $0.8958(2)$ & 0.5608 & $0.0855(15)$ \\
\hline $\mathrm{H} 21 \mathrm{~A}$ & 0.7549 & 0.9423 & 0.5386 & $0.128^{*}$ \\
\hline $\mathrm{H} 21 \mathrm{~B}$ & 0.8395 & 0.8811 & 0.5210 & $0.128^{*}$ \\
\hline $\mathrm{H} 21 \mathrm{C}$ & 0.8183 & 0.8939 & 0.6431 & $0.128 *$ \\
\hline
\end{tabular}




\begin{tabular}{|c|c|c|c|c|}
\hline $\mathrm{C} 22$ & $1.0168(3)$ & $0.7020(2)$ & $1.1400(3)$ & $0.0445(8)$ \\
\hline $\mathrm{H} 22 \mathrm{~A}$ & 1.0756 & 0.7131 & 1.0954 & $0.053 *$ \\
\hline $\mathrm{C} 23$ & $1.0425(4)$ & $0.7513(2)$ & $1.2420(3)$ & $0.0535(9)$ \\
\hline $\mathrm{H} 23 \mathrm{~A}$ & 1.0095 & 0.7962 & 1.2135 & $0.064 *$ \\
\hline H23B & 1.1320 & 0.7561 & 1.2734 & $0.064 *$ \\
\hline $\mathrm{C} 24$ & $0.9880(3)$ & $0.7304(2)$ & $1.3406(3)$ & $0.0502(9)$ \\
\hline $\mathrm{C} 25$ & $1.0223(3)$ & $0.6553(2)$ & $1.3694(3)$ & $0.0476(9)$ \\
\hline $\mathrm{H} 25 \mathrm{~A}$ & 1.1136 & 0.6529 & 1.3941 & $0.057 *$ \\
\hline $\mathrm{C} 26$ & 0.9787 (3) & $0.6105(2)$ & $1.2625(3)$ & $0.0473(8)$ \\
\hline $\mathrm{H} 26 \mathrm{~A}$ & 0.8881 & 0.6145 & 1.2312 & $0.057^{*}$ \\
\hline $\mathrm{C} 27$ & $1.0414(5)$ & $0.7765(3)$ & $1.4463(3)$ & $0.0729(12)$ \\
\hline $\mathrm{H} 27 \mathrm{~A}$ & 1.0196 & 0.8236 & 1.4260 & $0.109^{*}$ \\
\hline H27B & 1.1305 & 0.7719 & 1.4711 & $0.109^{*}$ \\
\hline $\mathrm{H} 27 \mathrm{C}$ & 1.0075 & 0.7629 & 1.5082 & $0.109 *$ \\
\hline $\mathrm{C} 28$ & $0.7942(5)$ & $0.7927(2)$ & $1.2621(4)$ & $0.0775(13)$ \\
\hline $\mathrm{H} 28 \mathrm{~A}$ & 0.8396 & 0.8315 & 1.3027 & $0.116^{*}$ \\
\hline H28B & 0.7113 & 0.7928 & 1.2707 & $0.116^{*}$ \\
\hline $\mathrm{H} 28 \mathrm{C}$ & 0.7900 & 0.7958 & 1.1813 & $0.116^{*}$ \\
\hline $\mathrm{C} 29$ & $1.0136(5)$ & $0.5356(2)$ & $1.2872(4)$ & $0.0731(12)$ \\
\hline $\mathrm{H} 29 \mathrm{~A}$ & 0.9827 & 0.5092 & 1.2175 & $0.110^{*}$ \\
\hline $\mathrm{H} 29 \mathrm{~B}$ & 0.9776 & 0.5187 & 1.3457 & $0.110^{*}$ \\
\hline $\mathrm{H} 29 \mathrm{C}$ & 1.1027 & 0.5314 & 1.3140 & $0.110^{*}$ \\
\hline C30 & $0.6344(3)$ & $0.52944(18)$ & $1.0432(3)$ & $0.0379(7)$ \\
\hline H30A & 0.6512 & 0.5700 & 1.0939 & $0.046^{*}$ \\
\hline C31 & $0.6781(3)$ & $0.4524(2)$ & $1.2038(3)$ & $0.0551(10)$ \\
\hline H31A & 0.6926 & 0.4917 & 1.2571 & $0.066^{*}$ \\
\hline C32 & $0.5423(3)$ & 0.4309 (2) & $1.1766(3)$ & $0.0571(10)$ \\
\hline $\mathrm{H} 32 \mathrm{~A}$ & 0.5225 & 0.4206 & 1.2486 & $0.069^{*}$ \\
\hline H32B & 0.5295 & 0.3893 & 1.1301 & $0.069^{*}$ \\
\hline C33 & $0.4542(3)$ & 0.48677 (19) & 1.1117 (3) & $0.0414(8)$ \\
\hline H33A & 0.4631 & 0.5260 & 1.1647 & $0.050 *$ \\
\hline C34 & $0.4951(3)$ & $0.51140(18)$ & $1.0086(3)$ & $0.0379(7)$ \\
\hline H34A & 0.4807 & 0.4741 & 0.9512 & $0.046^{*}$ \\
\hline $\mathrm{C} 35$ & 0.7669 (4) & $0.3950(3)$ & $1.2574(4)$ & $0.0831(14)$ \\
\hline H35A & 0.8512 & 0.4112 & 1.2729 & $0.125^{*}$ \\
\hline H35B & 0.7546 & 0.3567 & 1.2047 & $0.125^{*}$ \\
\hline $\mathrm{H} 35 \mathrm{C}$ & 0.7515 & 0.3806 & 1.3286 & $0.125^{*}$ \\
\hline C36 & $0.2757(4)$ & $0.4494(2)$ & $1.1756(4)$ & $0.0681(12)$ \\
\hline H36A & 0.1863 & 0.4466 & 1.1525 & $0.102 *$ \\
\hline H36B & 0.3022 & 0.4837 & 1.2352 & $0.102 *$ \\
\hline $\mathrm{H} 36 \mathrm{C}$ & 0.3101 & 0.4055 & 1.2049 & $0.102 *$ \\
\hline C37 & $0.2853(4)$ & $0.4155(2)$ & $0.9868(3)$ & $0.0574(10)$ \\
\hline H37A & 0.3259 & 0.3731 & 1.0169 & $0.086^{*}$ \\
\hline H37B & 0.3109 & 0.4293 & 0.9202 & $0.086^{*}$ \\
\hline $\mathrm{H} 37 \mathrm{C}$ & 0.1966 & 0.4090 & 0.9648 & $0.086^{*}$ \\
\hline $\mathrm{S} 1$ & $0.25601(10)$ & $0.59971(10)$ & $0.65505(9)$ & $0.0772(4)$ \\
\hline $\mathrm{O} 14$ & 0.3849 (3) & $0.5796(2)$ & $0.7224(3)$ & 0.0901 (11) \\
\hline C38 & $0.2103(6)$ & 0.6649 & $0.7334(6)$ & $0.118(2)$ \\
\hline
\end{tabular}




$\begin{array}{llllll}\text { H38A } & 0.2281 & 0.6514 & 0.8133 & 0.177^{*} & \\ \text { H38B } & 0.1224 & 0.6729 & 0.7026 & 0.177^{*} & \\ \text { H38C } & 0.2550 & 0.7063 & 0.7276 & 0.177^{*} & \\ \text { C39 } & 0.1537(5) & 0.5351(4) & 0.6804(5) & 0.1078(19) & \\ \text { H39A } & 0.1596 & 0.4947 & 0.6361 & 0.162^{*} & \\ \text { H39B } & 0.0696 & 0.5520 & 0.6575 & 0.162^{*} & \\ \text { H39C } & 0.1767 & 0.5237 & 0.7615 & 0.162^{*} & \\ \text { S2 } & 0.32316(11) & 0.78019(6) & 0.13850(11) & 0.0831(4) & 0.482(4) \\ \text { O15 } & 0.3312(7) & 0.7602(4) & 0.2610(4) & 0.0988(12) & 0.482(4) \\ \text { C40 } & 0.3945(18) & 0.7122(7) & 0.0884(13) & 0.129(3) & 0.482(4) \\ \text { H40D } & 0.3430 & 0.6719 & 0.0804 & 0.193^{*} & 0.482(4) \\ \text { H40E } & 0.4741 & 0.7031 & 0.1426 & 0.193^{*} & 0.482(4) \\ \text { H40F } & 0.4060 & 0.7239 & 0.0143 & 0.193^{*} & 0.482(4) \\ \text { C41 } & 0.4354(12) & 0.8434(6) & 0.1485(12) & 0.126(3) & 0.482(4) \\ \text { H41A } & 0.4095 & 0.8850 & 0.1784 & 0.189^{*} & 0.482(4) \\ \text { H41B } & 0.4456 & 0.8521 & 0.0729 & 0.189^{*} & 0.482(4) \\ \text { H41C } & 0.5131 & 0.8282 & 0.1997 & 0.189^{*} & 0.482(4) \\ \text { S2' } & 0.32316(11) & 0.78019(6) & 0.13850(11) & 0.0831(4) & 0.518(4) \\ \text { O15' } & 0.3821(7) & 0.8140(3) & 0.2530(4) & 0.0988(12) & 0.518(4) \\ \text { C40' } & 0.3841(18) & 0.6976(5) & 0.1450(13) & 0.129(3) & 0.518(4) \\ \text { H40A } & 0.3503 & 0.6696 & 0.1947 & 0.193^{*} & 0.518(4) \\ \text { H40B } & 0.4732 & 0.6995 & 0.1752 & 0.193^{*} & 0.518(4) \\ \text { H40C } & 0.3626 & 0.6780 & 0.0685 & 0.193^{*} & 0.518(4) \\ \text { C41' } & 0.4084(12) & 0.8139(7) & 0.0506(10) & 0.126(3) & 0.518(4) \\ \text { H41D } & 0.3887 & 0.8619 & 0.0367 & 0.189^{*} & 0.518(4) \\ \text { H41E } & 0.3878 & 0.7896 & -0.0219 & 0.189^{*} & 0.518(4) \\ \text { H41F } & 0.4958 & 0.8089 & 0.0885 & 0.189^{*} & 0.518(4) \\ & & & & & \end{array}$

Atomic displacement parameters $\left(\AA^{2}\right)$

\begin{tabular}{lllllll}
\hline & $U^{11}$ & $U^{22}$ & $U^{33}$ & $U^{12}$ & $U^{13}$ & $U^{23}$ \\
\hline O1 & $0.0607(16)$ & $0.100(2)$ & $0.0466(14)$ & $0.0232(16)$ & $0.0213(13)$ & $0.0065(15)$ \\
O3 & $0.0416(12)$ & $0.0527(14)$ & $0.0337(11)$ & $0.0013(10)$ & $0.0155(10)$ & $0.0010(10)$ \\
O5 & $0.0376(12)$ & $0.0434(12)$ & $0.0368(11)$ & $-0.0056(10)$ & $0.0174(9)$ & $0.0028(10)$ \\
O6 & $0.0447(13)$ & $0.0531(14)$ & $0.0593(15)$ & $-0.0022(12)$ & $0.0272(11)$ & $-0.0008(12)$ \\
O7W & $0.0553(17)$ & $0.089(2)$ & $0.0660(18)$ & $0.0140(16)$ & $0.0195(14)$ & $0.0020(17)$ \\
O8W & $0.098(7)$ & $0.107(7)$ & $0.108(7)$ & $0.008(5)$ & $0.018(5)$ & $0.035(5)$ \\
O9 & $0.133(3)$ & $0.071(2)$ & $0.085(2)$ & $-0.027(2)$ & $0.026(2)$ & $-0.0360(19)$ \\
O11 & $0.0719(18)$ & $0.0600(17)$ & $0.0573(16)$ & $-0.0040(13)$ & $0.0328(14)$ & $0.0000(13)$ \\
O12 & $0.079(2)$ & $0.096(2)$ & $0.0426(14)$ & $0.0130(19)$ & $-0.0045(13)$ & $-0.0086(15)$ \\
O13 & $0.0442(13)$ & $0.0525(14)$ & $0.0348(11)$ & $0.0021(11)$ & $0.0095(10)$ & $-0.0015(10)$ \\
O24 & $0.0551(15)$ & $0.0602(16)$ & $0.0537(14)$ & $0.0110(13)$ & $0.0187(12)$ & $0.0153(13)$ \\
O25 & $0.0606(16)$ & $0.0774(19)$ & $0.0468(14)$ & $0.0079(14)$ & $0.0230(12)$ & $0.0203(13)$ \\
O26 & $0.0482(13)$ & $0.0596(16)$ & $0.0418(13)$ & $0.0092(11)$ & $0.0155(11)$ & $0.0054(11)$ \\
O30 & $0.0460(14)$ & $0.0614(16)$ & $0.0490(14)$ & $0.0072(12)$ & $0.0185(11)$ & $0.0173(12)$ \\
O34 & $0.0453(13)$ & $0.0538(15)$ & $0.0624(15)$ & $0.0109(11)$ & $0.0254(12)$ & $0.0157(12)$ \\
N33 & $0.0462(16)$ & $0.0517(18)$ & $0.0483(17)$ & $-0.0118(14)$ & $0.0235(13)$ & $-0.0027(14)$ \\
C1 & $0.0444(19)$ & $0.0345(18)$ & $0.0403(17)$ & $-0.0037(15)$ & $0.0162(15)$ & $0.0054(14)$
\end{tabular}




\begin{tabular}{|c|c|c|c|c|c|c|}
\hline $\mathrm{C} 2$ & $0.0419(17)$ & $0.0390(18)$ & $0.0359(16)$ & $-0.0019(14)$ & 0.0109 (14) & $-0.0022(14)$ \\
\hline $\mathrm{C} 3$ & $0.0350(16)$ & $0.0338(16)$ & $0.0323(15)$ & $0.0028(13)$ & $0.0106(13)$ & $0.0019(13)$ \\
\hline $\mathrm{C} 4$ & $0.0318(16)$ & 0.0384 (19) & $0.0396(17)$ & $0.0003(13)$ & $0.0115(13)$ & $0.0022(14)$ \\
\hline $\mathrm{C} 5$ & $0.0293(15)$ & $0.0380(17)$ & $0.0377(16)$ & $-0.0026(13)$ & 0.0119 (13) & $0.0056(14)$ \\
\hline C6 & $0.0425(19)$ & $0.0395(19)$ & $0.056(2)$ & $-0.0014(15)$ & $0.0229(16)$ & $0.0039(16)$ \\
\hline $\mathrm{C} 7$ & $0.053(2)$ & $0.046(2)$ & $0.048(2)$ & $-0.0054(16)$ & $0.0236(16)$ & $-0.0068(16)$ \\
\hline $\mathrm{C} 8$ & 0.075 & $0.038(2)$ & $0.058(2)$ & $-0.0027(18)$ & $0.036(2)$ & $-0.0088(17)$ \\
\hline C9 & 0.083 & $0.054(3)$ & $0.051(2)$ & $-0.020(2)$ & $0.032(2)$ & $-0.015(2)$ \\
\hline C10 & $0.056(2)$ & $0.071(3)$ & $0.0400(18)$ & -0.017 (2) & $0.0141(16)$ & $-0.0113(18)$ \\
\hline C11 & $0.0454(19)$ & $0.061(2)$ & 0.0383 (19) & $-0.0096(17)$ & $0.0137(15)$ & $-0.0058(17)$ \\
\hline $\mathrm{C} 12$ & $0.048(2)$ & $0.060(2)$ & 0.0412 (19) & $-0.0007(18)$ & $0.0044(16)$ & $-0.0028(17)$ \\
\hline $\mathrm{C} 13$ & 0.059 (2) & $0.048(2)$ & $0.0343(17)$ & $-0.0002(17)$ & $0.0096(15)$ & $0.0023(15)$ \\
\hline C14 & $0.063(2)$ & $0.053(2)$ & $0.052(2)$ & $-0.0158(19)$ & $0.0111(18)$ & $0.0087(18)$ \\
\hline $\mathrm{C} 15$ & $0.040(2)$ & $0.042(2)$ & $0.096(3)$ & $0.0038(16)$ & $0.028(2)$ & $0.006(2)$ \\
\hline C16 & $0.062(2)$ & $0.043(2)$ & $0.073(3)$ & $0.0062(18)$ & $0.027(2)$ & $0.0029(19)$ \\
\hline $\mathrm{C} 17$ & $0.127(4)$ & $0.081(3)$ & 0.093 (4) & $0.023(3)$ & $0.062(3)$ & $-0.019(3)$ \\
\hline $\mathrm{C} 18$ & $0.082(3)$ & $0.104(4)$ & $0.061(3)$ & -0.033 & $-0.001(2)$ & $-0.017(3)$ \\
\hline C19 & $0.049(2)$ & $0.092(3)$ & 0.080 & $0.004(2)$ & $0.014(2)$ & -0.011 \\
\hline $\mathrm{C} 20$ & $0.097(3)$ & $0.055(2)$ & $0.047(2)$ & $0.000(2)$ & $0.006(2)$ & 0.0005 (19) \\
\hline $\mathrm{C} 21$ & $0.144(5)$ & 0.050 & $0.072(3)$ & $-0.011(3)$ & $0.044(3)$ & $-0.002(2)$ \\
\hline $\mathrm{C} 22$ & $0.0383(18)$ & $0.058(2)$ & $0.0389(17)$ & $-0.0050(16)$ & $0.0140(15)$ & $0.0011(16)$ \\
\hline $\mathrm{C} 23$ & $0.058(2)$ & $0.063(2)$ & $0.0410(19)$ & $-0.0133(19)$ & $0.0151(16)$ & $-0.0005(17)$ \\
\hline $\mathrm{C} 24$ & $0.060(2)$ & $0.055(2)$ & $0.0375(18)$ & $-0.0081(18)$ & $0.0171(16)$ & $-0.0008(16)$ \\
\hline $\mathrm{C} 25$ & $0.0391(18)$ & $0.069(2)$ & $0.0361(17)$ & $0.0025(17)$ & $0.0121(14)$ & $0.0079(17)$ \\
\hline $\mathrm{C} 26$ & $0.0414(18)$ & $0.056(2)$ & 0.0440 (19) & $0.0052(17)$ & $0.0105(15)$ & 0.0089 (17) \\
\hline $\mathrm{C} 27$ & $0.104(3)$ & 0.075 & $0.045(2)$ & $-0.019(3)$ & $0.028(2)$ & $-0.014(2)$ \\
\hline $\mathrm{C} 28$ & $0.098(3)$ & $0.067(3)$ & $0.068(3)$ & $0.030(3)$ & $0.025(3)$ & $0.014(2)$ \\
\hline $\mathrm{C} 29$ & 0.107 (4) & $0.049(2)$ & $0.064(3)$ & $0.015(2)$ & $0.025(2)$ & $0.010(2)$ \\
\hline $\mathrm{C} 30$ & $0.0440(18)$ & $0.0349(18)$ & $0.0380(17)$ & $-0.0009(14)$ & $0.0166(14)$ & $0.0002(14)$ \\
\hline C31 & $0.053(2)$ & $0.069(3)$ & $0.045(2)$ & $-0.0018(19)$ & $0.0162(17)$ & $0.0171(18)$ \\
\hline $\mathrm{C} 32$ & $0.063(2)$ & $0.062(2)$ & $0.052(2)$ & $-0.008(2)$ & $0.0256(19)$ & $0.0126(19)$ \\
\hline $\mathrm{C} 33$ & $0.0416(18)$ & $0.048(2)$ & $0.0362(17)$ & $-0.0073(15)$ & $0.0142(14)$ & $-0.0032(15)$ \\
\hline C34 & $0.0397(17)$ & $0.0386(18)$ & $0.0393(17)$ & $-0.0004(15)$ & $0.0174(14)$ & $0.0006(15)$ \\
\hline C35 & $0.074(3)$ & $0.094(4)$ & $0.078(3)$ & $0.005(3)$ & $0.016(3)$ & $0.041(3)$ \\
\hline C36 & $0.072(3)$ & $0.082(3)$ & $0.065(3)$ & $-0.024(2)$ & $0.043(2)$ & $-0.012(2)$ \\
\hline C37 & $0.055(2)$ & $0.055(2)$ & $0.063(2)$ & $-0.0121(18)$ & $0.0181(19)$ & $-0.0080(19)$ \\
\hline $\mathrm{S} 1$ & $0.0550(6)$ & $0.1259(11)$ & $0.0512(6)$ & $0.0139(7)$ & $0.0158(5)$ & $0.0244(6)$ \\
\hline $\mathrm{O} 14$ & $0.0610(18)$ & $0.141(3)$ & 0.0719 (19) & $0.027(2)$ & $0.0256(15)$ & $0.024(2)$ \\
\hline C38 & $0.112(5)$ & $0.118(5)$ & $0.116(5)$ & $0.045(4)$ & $0.019(4)$ & 0.007 (4) \\
\hline C39 & $0.086(4)$ & $0.140(5)$ & $0.096(4)$ & 0.000 & $0.025(3)$ & $0.023(4)$ \\
\hline $\mathrm{S} 2$ & $0.0556(6)$ & $0.1101(10)$ & $0.0765(7)$ & $0.0036(6)$ & $0.0067(5)$ & $0.0220(7)$ \\
\hline O15 & 0.104 (4) & $0.108(3)$ & $0.0781(11)$ & $-0.003(2)$ & $0.014(2)$ & $0.0190(15)$ \\
\hline $\mathrm{C} 40$ & $0.160(7)$ & $0.121(3)$ & $0.103(8)$ & $0.032(5)$ & $0.032(7)$ & $0.010(2)$ \\
\hline $\mathrm{C} 41$ & $0.136(7)$ & $0.148(5)$ & $0.106(4)$ & $-0.052(4)$ & $0.054(4)$ & $-0.014(3)$ \\
\hline $\mathrm{S} 2^{\prime}$ & $0.0556(6)$ & $0.1101(10)$ & $0.0765(7)$ & $0.0036(6)$ & $0.0067(5)$ & $0.0220(7)$ \\
\hline O15' & $0.104(4)$ & $0.108(3)$ & $0.0781(11)$ & $-0.003(2)$ & $0.014(2)$ & $0.0190(15)$ \\
\hline $\mathrm{C} 40^{\prime}$ & $0.160(7)$ & $0.121(3)$ & $0.103(8)$ & $0.032(5)$ & $0.032(7)$ & $0.010(2)$ \\
\hline $\mathrm{C} 41^{\prime}$ & $0.136(7)$ & $0.148(5)$ & $0.106(4)$ & $-0.052(4)$ & $0.054(4)$ & -0.014 \\
\hline
\end{tabular}


Geometric parameters $\left(\AA,{ }^{\circ}\right)$

\begin{tabular}{|c|c|c|c|}
\hline $\mathrm{O} 1-\mathrm{C} 1$ & 1.197 (4) & C19-H19A & 0.9600 \\
\hline $\mathrm{O} 3-\mathrm{C} 22$ & $1.416(4)$ & C19-H19B & 0.9600 \\
\hline $\mathrm{O} 3-\mathrm{C} 3$ & $1.440(4)$ & $\mathrm{C} 19-\mathrm{H} 19 \mathrm{C}$ & 0.9600 \\
\hline $\mathrm{O} 5-\mathrm{C} 30$ & $1.383(3)$ & $\mathrm{C} 20-\mathrm{C} 21$ & $1.513(7)$ \\
\hline $\mathrm{O} 5-\mathrm{C} 5$ & $1.454(3)$ & $\mathrm{C} 20-\mathrm{H} 20 \mathrm{~A}$ & 0.9700 \\
\hline $\mathrm{O} 6-\mathrm{C} 6$ & $1.445(4)$ & $\mathrm{C} 20-\mathrm{H} 20 \mathrm{~B}$ & 0.9700 \\
\hline O6-H6A & 0.8200 & $\mathrm{C} 21-\mathrm{H} 21 \mathrm{~A}$ & 0.9600 \\
\hline $\mathrm{O} 7 \mathrm{~W}-\mathrm{H} 7 \mathrm{C}$ & $0.845(10)$ & $\mathrm{C} 21-\mathrm{H} 21 \mathrm{~B}$ & 0.9600 \\
\hline $\mathrm{O} 7 \mathrm{~W}-\mathrm{H} 7 \mathrm{D}$ & $0.841(10)$ & $\mathrm{C} 21-\mathrm{H} 21 \mathrm{C}$ & 0.9600 \\
\hline $\mathrm{O} 9-\mathrm{C} 9$ & $1.210(5)$ & $\mathrm{C} 22-\mathrm{C} 23$ & $1.517(5)$ \\
\hline $\mathrm{O} 11-\mathrm{C} 11$ & $1.434(4)$ & $\mathrm{C} 22-\mathrm{H} 22 \mathrm{~A}$ & 0.9800 \\
\hline $\mathrm{O} 11-\mathrm{H} 11 \mathrm{~B}$ & 0.8200 & $\mathrm{C} 23-\mathrm{C} 24$ & $1.529(5)$ \\
\hline $\mathrm{O} 12-\mathrm{C} 12$ & $1.436(4)$ & $\mathrm{C} 23-\mathrm{H} 23 \mathrm{~A}$ & 0.9700 \\
\hline $\mathrm{O} 12-\mathrm{H} 12 \mathrm{~A}$ & 0.8200 & $\mathrm{C} 23-\mathrm{H} 23 \mathrm{~B}$ & 0.9700 \\
\hline $\mathrm{O} 13-\mathrm{C} 1$ & $1.345(4)$ & $\mathrm{C} 24-\mathrm{C} 25$ & $1.523(5)$ \\
\hline $\mathrm{O} 13-\mathrm{C} 13$ & 1.464 (4) & $\mathrm{C} 24-\mathrm{C} 27$ & $1.530(5)$ \\
\hline $\mathrm{O} 24-\mathrm{C} 28$ & $1.407(5)$ & $\mathrm{C} 25-\mathrm{C} 26$ & $1.512(5)$ \\
\hline $\mathrm{O} 24-\mathrm{C} 24$ & $1.425(4)$ & $\mathrm{C} 25-\mathrm{H} 25 \mathrm{~A}$ & 0.9800 \\
\hline $\mathrm{O} 25-\mathrm{C} 25$ & $1.419(4)$ & $\mathrm{C} 26-\mathrm{C} 29$ & $1.513(5)$ \\
\hline $\mathrm{O} 25-\mathrm{H} 25 \mathrm{~B}$ & 0.8200 & $\mathrm{C} 26-\mathrm{H} 26 \mathrm{~A}$ & 0.9800 \\
\hline $\mathrm{O} 26-\mathrm{C} 22$ & $1.421(4)$ & $\mathrm{C} 27-\mathrm{H} 27 \mathrm{~A}$ & 0.9600 \\
\hline $\mathrm{O} 26-\mathrm{C} 26$ & $1.438(4)$ & $\mathrm{C} 27-\mathrm{H} 27 \mathrm{~B}$ & 0.9600 \\
\hline $\mathrm{O} 30-\mathrm{C} 30$ & $1.414(4)$ & $\mathrm{C} 27-\mathrm{H} 27 \mathrm{C}$ & 0.9600 \\
\hline $\mathrm{O} 30-\mathrm{C} 31$ & $1.450(4)$ & $\mathrm{C} 28-\mathrm{H} 28 \mathrm{~A}$ & 0.9600 \\
\hline $\mathrm{O} 34-\mathrm{C} 34$ & $1.415(4)$ & $\mathrm{C} 28-\mathrm{H} 28 \mathrm{~B}$ & 0.9600 \\
\hline $\mathrm{O} 34-\mathrm{H} 34 \mathrm{~B}$ & 0.8200 & $\mathrm{C} 28-\mathrm{H} 28 \mathrm{C}$ & 0.9600 \\
\hline N33-C37 & $1.448(5)$ & $\mathrm{C} 29-\mathrm{H} 29 \mathrm{~A}$ & 0.9600 \\
\hline N33-C36 & $1.470(4)$ & $\mathrm{C} 29-\mathrm{H} 29 \mathrm{~B}$ & 0.9600 \\
\hline N33-C33 & $1.490(4)$ & $\mathrm{C} 29-\mathrm{H} 29 \mathrm{C}$ & 0.9600 \\
\hline $\mathrm{C} 1-\mathrm{C} 2$ & $1.519(4)$ & $\mathrm{C} 30-\mathrm{C} 34$ & $1.534(4)$ \\
\hline $\mathrm{C} 2-\mathrm{C} 14$ & $1.514(5)$ & $\mathrm{C} 30-\mathrm{H} 30 \mathrm{~A}$ & 0.9800 \\
\hline $\mathrm{C} 2-\mathrm{C} 3$ & $1.542(4)$ & $\mathrm{C} 31-\mathrm{C} 35$ & $1.511(6)$ \\
\hline $\mathrm{C} 2-\mathrm{H} 2 \mathrm{~A}$ & 0.9800 & $\mathrm{C} 31-\mathrm{C} 32$ & $1.518(5)$ \\
\hline $\mathrm{C} 3-\mathrm{C} 4$ & $1.544(4)$ & $\mathrm{C} 31-\mathrm{H} 31 \mathrm{~A}$ & 0.9800 \\
\hline $\mathrm{C} 3-\mathrm{H} 3 \mathrm{~A}$ & 0.9800 & $\mathrm{C} 32-\mathrm{C} 33$ & $1.523(5)$ \\
\hline $\mathrm{C} 4-\mathrm{C} 15$ & $1.546(5)$ & $\mathrm{C} 32-\mathrm{H} 32 \mathrm{~A}$ & 0.9700 \\
\hline $\mathrm{C} 4-\mathrm{C} 5$ & $1.548(4)$ & $\mathrm{C} 32-\mathrm{H} 32 \mathrm{~B}$ & 0.9700 \\
\hline $\mathrm{C} 4-\mathrm{H} 4 \mathrm{~A}$ & 0.9800 & $\mathrm{C} 33-\mathrm{C} 34$ & $1.511(4)$ \\
\hline $\mathrm{C} 5-\mathrm{C} 6$ & $1.558(4)$ & $\mathrm{C} 33-\mathrm{H} 33 \mathrm{~A}$ & 0.9800 \\
\hline $\mathrm{C} 5-\mathrm{H} 5 \mathrm{~A}$ & 0.9800 & $\mathrm{C} 34-\mathrm{H} 34 \mathrm{~A}$ & 0.9800 \\
\hline $\mathrm{C} 6-\mathrm{C} 16$ & $1.516(5)$ & C35-H35A & 0.9600 \\
\hline $\mathrm{C} 6-\mathrm{C} 7$ & $1.548(5)$ & C35-H35B & 0.9600 \\
\hline $\mathrm{C} 7-\mathrm{C} 8$ & $1.542(5)$ & $\mathrm{C} 35-\mathrm{H} 35 \mathrm{C}$ & 0.9600 \\
\hline C7-H7A & 0.9700 & $\mathrm{C} 36-\mathrm{H} 36 \mathrm{~A}$ & 0.9600 \\
\hline C7-H7B & 0.9700 & C36-H36B & 0.9600 \\
\hline $\mathrm{C} 8-\mathrm{C} 9$ & $1.512(6)$ & $\mathrm{C} 36-\mathrm{H} 36 \mathrm{C}$ & 0.9600 \\
\hline
\end{tabular}




\begin{tabular}{|c|c|c|c|}
\hline $\mathrm{C} 8-\mathrm{C} 17$ & $1.542(6)$ & C37-H37A & 0.9600 \\
\hline $\mathrm{C} 8-\mathrm{H} 8 \mathrm{~A}$ & 0.9800 & C37-H37B & 0.9600 \\
\hline $\mathrm{C} 9-\mathrm{C} 10$ & $1.532(6)$ & $\mathrm{C} 37-\mathrm{H} 37 \mathrm{C}$ & 0.9600 \\
\hline $\mathrm{C} 10-\mathrm{C} 11$ & $1.537(5)$ & $\mathrm{S} 1-\mathrm{O} 14$ & $1.493(3)$ \\
\hline $\mathrm{C} 10-\mathrm{C} 18$ & $1.549(5)$ & $\mathrm{S} 1-\mathrm{C} 38$ & $1.735(6)$ \\
\hline $\mathrm{C} 10-\mathrm{H} 10 \mathrm{~A}$ & 0.9800 & $\mathrm{~S} 1-\mathrm{C} 39$ & $1.779(6)$ \\
\hline $\mathrm{C} 11-\mathrm{C} 12$ & $1.536(6)$ & $\mathrm{C} 38-\mathrm{H} 38 \mathrm{~A}$ & 0.9600 \\
\hline $\mathrm{C} 11-\mathrm{H} 11 \mathrm{~A}$ & 0.9800 & $\mathrm{C} 38-\mathrm{H} 38 \mathrm{~B}$ & 0.9600 \\
\hline $\mathrm{C} 12-\mathrm{C} 19$ & $1.514(5)$ & $\mathrm{C} 38-\mathrm{H} 38 \mathrm{C}$ & 0.9600 \\
\hline $\mathrm{C} 12-\mathrm{C} 13$ & $1.544(5)$ & C39-H39A & 0.9600 \\
\hline $\mathrm{C} 13-\mathrm{C} 20$ & $1.530(6)$ & C $39-\mathrm{H} 39 \mathrm{~B}$ & 0.9600 \\
\hline C13-H13A & 0.9800 & $\mathrm{C} 39-\mathrm{H} 39 \mathrm{C}$ & 0.9600 \\
\hline $\mathrm{C} 14-\mathrm{H} 14 \mathrm{~A}$ & 0.9600 & $\mathrm{~S} 2-\mathrm{O} 15$ & $1.498(5)$ \\
\hline C14-H14B & 0.9600 & $\mathrm{~S} 2-\mathrm{C} 41$ & $1.734(6)$ \\
\hline $\mathrm{C} 14-\mathrm{H} 14 \mathrm{C}$ & 0.9600 & $\mathrm{~S} 2-\mathrm{C} 40$ & $1.734(6)$ \\
\hline C15-H15A & 0.9600 & $\mathrm{C} 40-\mathrm{H} 40 \mathrm{D}$ & 0.9600 \\
\hline C15-H15B & 0.9600 & $\mathrm{C} 40-\mathrm{H} 40 \mathrm{E}$ & 0.9600 \\
\hline $\mathrm{C} 15-\mathrm{H} 15 \mathrm{C}$ & 0.9600 & $\mathrm{C} 40-\mathrm{H} 40 \mathrm{~F}$ & 0.9600 \\
\hline $\mathrm{C} 16-\mathrm{H} 16 \mathrm{~A}$ & 0.9600 & $\mathrm{C} 41-\mathrm{H} 41 \mathrm{~A}$ & 0.9600 \\
\hline C16-H16B & 0.9600 & $\mathrm{C} 41-\mathrm{H} 41 \mathrm{~B}$ & 0.9600 \\
\hline $\mathrm{C} 16-\mathrm{H} 16 \mathrm{C}$ & 0.9600 & $\mathrm{C} 41-\mathrm{H} 41 \mathrm{C}$ & 0.9600 \\
\hline C17-H17A & 0.9600 & $\mathrm{C} 40^{\prime}-\mathrm{H} 40 \mathrm{~A}$ & 0.9600 \\
\hline C17-H17B & 0.9600 & $\mathrm{C} 40^{\prime}-\mathrm{H} 40 \mathrm{~B}$ & 0.9600 \\
\hline $\mathrm{C} 17-\mathrm{H} 17 \mathrm{C}$ & 0.9600 & $\mathrm{C} 40^{\prime}-\mathrm{H} 40 \mathrm{C}$ & 0.9600 \\
\hline $\mathrm{C} 18-\mathrm{H} 18 \mathrm{~A}$ & 0.9600 & $\mathrm{C} 41^{\prime}-\mathrm{H} 41 \mathrm{D}$ & 0.9600 \\
\hline $\mathrm{C} 18-\mathrm{H} 18 \mathrm{~B}$ & 0.9600 & $\mathrm{C} 41^{\prime}-\mathrm{H} 41 \mathrm{E}$ & 0.9600 \\
\hline $\mathrm{C} 18-\mathrm{H} 18 \mathrm{C}$ & 0.9600 & $\mathrm{C} 41^{\prime}-\mathrm{H} 41 \mathrm{~F}$ & 0.9600 \\
\hline $\mathrm{C} 22-\mathrm{O} 3-\mathrm{C} 3$ & $116.0(2)$ & $\mathrm{H} 21 \mathrm{~B}-\mathrm{C} 21-\mathrm{H} 21 \mathrm{C}$ & 109.5 \\
\hline $\mathrm{C} 30-\mathrm{O} 5-\mathrm{C} 5$ & $120.3(2)$ & $\mathrm{O} 3-\mathrm{C} 22-\mathrm{O} 26$ & $111.6(3)$ \\
\hline $\mathrm{C} 6-\mathrm{O} 6-\mathrm{H} 6 \mathrm{~A}$ & 109.5 & $\mathrm{O} 3-\mathrm{C} 22-\mathrm{C} 23$ & $110.7(3)$ \\
\hline $\mathrm{H} 7 \mathrm{C}-\mathrm{O} 7 \mathrm{~W}-\mathrm{H} 7 \mathrm{D}$ & $106(4)$ & $\mathrm{O} 26-\mathrm{C} 22-\mathrm{C} 23$ & $112.1(3)$ \\
\hline $\mathrm{C} 11-\mathrm{O} 11-\mathrm{H} 11 \mathrm{~B}$ & 109.5 & $\mathrm{O} 3-\mathrm{C} 22-\mathrm{H} 22 \mathrm{~A}$ & 107.4 \\
\hline $\mathrm{C} 12-\mathrm{O} 12-\mathrm{H} 12 \mathrm{~A}$ & 109.5 & $\mathrm{O} 26-\mathrm{C} 22-\mathrm{H} 22 \mathrm{~A}$ & 107.4 \\
\hline $\mathrm{C} 1-\mathrm{O} 13-\mathrm{C} 13$ & $119.5(2)$ & $\mathrm{C} 23-\mathrm{C} 22-\mathrm{H} 22 \mathrm{~A}$ & 107.4 \\
\hline $\mathrm{C} 28-\mathrm{O} 24-\mathrm{C} 24$ & $118.0(3)$ & $\mathrm{C} 22-\mathrm{C} 23-\mathrm{C} 24$ & $115.6(3)$ \\
\hline $\mathrm{C} 25-\mathrm{O} 25-\mathrm{H} 25 \mathrm{~B}$ & 109.5 & $\mathrm{C} 22-\mathrm{C} 23-\mathrm{H} 23 \mathrm{~A}$ & 108.4 \\
\hline $\mathrm{C} 22-\mathrm{O} 26-\mathrm{C} 26$ & $115.4(3)$ & $\mathrm{C} 24-\mathrm{C} 23-\mathrm{H} 23 \mathrm{~A}$ & 108.4 \\
\hline $\mathrm{C} 30-\mathrm{O} 30-\mathrm{C} 31$ & $112.4(2)$ & $\mathrm{C} 22-\mathrm{C} 23-\mathrm{H} 23 \mathrm{~B}$ & 108.4 \\
\hline $\mathrm{C} 34-\mathrm{O} 34-\mathrm{H} 34 \mathrm{~B}$ & 109.5 & $\mathrm{C} 24-\mathrm{C} 23-\mathrm{H} 23 \mathrm{~B}$ & 108.4 \\
\hline C37-N33-C36 & $110.0(3)$ & $\mathrm{H} 23 \mathrm{~A}-\mathrm{C} 23-\mathrm{H} 23 \mathrm{~B}$ & 107.4 \\
\hline $\mathrm{C} 37-\mathrm{N} 33-\mathrm{C} 33$ & $114.9(3)$ & $\mathrm{O} 24-\mathrm{C} 24-\mathrm{C} 25$ & $104.5(3)$ \\
\hline $\mathrm{C} 36-\mathrm{N} 33-\mathrm{C} 33$ & $111.0(3)$ & $\mathrm{O} 24-\mathrm{C} 24-\mathrm{C} 23$ & $113.3(3)$ \\
\hline $\mathrm{O} 1-\mathrm{C} 1-\mathrm{O} 13$ & $123.8(3)$ & $\mathrm{C} 25-\mathrm{C} 24-\mathrm{C} 23$ & $107.4(3)$ \\
\hline $\mathrm{O} 1-\mathrm{C} 1-\mathrm{C} 2$ & $124.8(3)$ & $\mathrm{O} 24-\mathrm{C} 24-\mathrm{C} 27$ & $110.7(3)$ \\
\hline $\mathrm{O} 13-\mathrm{C} 1-\mathrm{C} 2$ & $111.4(3)$ & $\mathrm{C} 25-\mathrm{C} 24-\mathrm{C} 27$ & $110.9(3)$ \\
\hline $\mathrm{C} 14-\mathrm{C} 2-\mathrm{C} 1$ & $107.5(3)$ & $\mathrm{C} 23-\mathrm{C} 24-\mathrm{C} 27$ & $109.9(3)$ \\
\hline $\mathrm{C} 14-\mathrm{C} 2-\mathrm{C} 3$ & $112.2(3)$ & $\mathrm{O} 25-\mathrm{C} 25-\mathrm{C} 26$ & $111.9(3)$ \\
\hline
\end{tabular}




\begin{tabular}{|c|c|c|c|}
\hline $\mathrm{C} 1-\mathrm{C} 2-\mathrm{C} 3$ & $112.0(2)$ & $\mathrm{O} 25-\mathrm{C} 25-\mathrm{C} 24$ & $113.2(3)$ \\
\hline $\mathrm{C} 14-\mathrm{C} 2-\mathrm{H} 2 \mathrm{~A}$ & 108.4 & $\mathrm{C} 26-\mathrm{C} 25-\mathrm{C} 24$ & $110.9(3)$ \\
\hline $\mathrm{C} 1-\mathrm{C} 2-\mathrm{H} 2 \mathrm{~A}$ & 108.4 & $\mathrm{O} 25-\mathrm{C} 25-\mathrm{H} 25 \mathrm{~A}$ & 106.8 \\
\hline $\mathrm{C} 3-\mathrm{C} 2-\mathrm{H} 2 \mathrm{~A}$ & 108.4 & $\mathrm{C} 26-\mathrm{C} 25-\mathrm{H} 25 \mathrm{~A}$ & 106.8 \\
\hline $\mathrm{O} 3-\mathrm{C} 3-\mathrm{C} 2$ & $108.2(2)$ & $\mathrm{C} 24-\mathrm{C} 25-\mathrm{H} 25 \mathrm{~A}$ & 106.8 \\
\hline $\mathrm{O} 3-\mathrm{C} 3-\mathrm{C} 4$ & $108.5(2)$ & $\mathrm{O} 26-\mathrm{C} 26-\mathrm{C} 25$ & $109.9(3)$ \\
\hline $\mathrm{C} 2-\mathrm{C} 3-\mathrm{C} 4$ & $113.1(2)$ & $\mathrm{O} 26-\mathrm{C} 26-\mathrm{C} 29$ & $106.1(3)$ \\
\hline $\mathrm{O} 3-\mathrm{C} 3-\mathrm{H} 3 \mathrm{~A}$ & 109.0 & $\mathrm{C} 25-\mathrm{C} 26-\mathrm{C} 29$ & $112.4(3)$ \\
\hline $\mathrm{C} 2-\mathrm{C} 3-\mathrm{H} 3 \mathrm{~A}$ & 109.0 & $\mathrm{O} 26-\mathrm{C} 26-\mathrm{H} 26 \mathrm{~A}$ & 109.5 \\
\hline $\mathrm{C} 4-\mathrm{C} 3-\mathrm{H} 3 \mathrm{~A}$ & 109.0 & $\mathrm{C} 25-\mathrm{C} 26-\mathrm{H} 26 \mathrm{~A}$ & 109.5 \\
\hline $\mathrm{C} 3-\mathrm{C} 4-\mathrm{C} 15$ & $110.7(3)$ & $\mathrm{C} 29-\mathrm{C} 26-\mathrm{H} 26 \mathrm{~A}$ & 109.5 \\
\hline $\mathrm{C} 3-\mathrm{C} 4-\mathrm{C} 5$ & $112.2(2)$ & $\mathrm{C} 24-\mathrm{C} 27-\mathrm{H} 27 \mathrm{~A}$ & 109.5 \\
\hline $\mathrm{C} 15-\mathrm{C} 4-\mathrm{C} 5$ & $113.2(3)$ & $\mathrm{C} 24-\mathrm{C} 27-\mathrm{H} 27 \mathrm{~B}$ & 109.5 \\
\hline $\mathrm{C} 3-\mathrm{C} 4-\mathrm{H} 4 \mathrm{~A}$ & 106.8 & $\mathrm{H} 27 \mathrm{~A}-\mathrm{C} 27-\mathrm{H} 27 \mathrm{~B}$ & 109.5 \\
\hline $\mathrm{C} 15-\mathrm{C} 4-\mathrm{H} 4 \mathrm{~A}$ & 106.8 & $\mathrm{C} 24-\mathrm{C} 27-\mathrm{H} 27 \mathrm{C}$ & 109.5 \\
\hline $\mathrm{C} 5-\mathrm{C} 4-\mathrm{H} 4 \mathrm{~A}$ & 106.8 & $\mathrm{H} 27 \mathrm{~A}-\mathrm{C} 27-\mathrm{H} 27 \mathrm{C}$ & 109.5 \\
\hline $\mathrm{O} 5-\mathrm{C} 5-\mathrm{C} 4$ & $111.1(2)$ & $\mathrm{H} 27 \mathrm{~B}-\mathrm{C} 27-\mathrm{H} 27 \mathrm{C}$ & 109.5 \\
\hline $\mathrm{O} 5-\mathrm{C} 5-\mathrm{C} 6$ & $103.0(2)$ & $\mathrm{O} 24-\mathrm{C} 28-\mathrm{H} 28 \mathrm{~A}$ & 109.5 \\
\hline $\mathrm{C} 4-\mathrm{C} 5-\mathrm{C} 6$ & $113.9(2)$ & $\mathrm{O} 24-\mathrm{C} 28-\mathrm{H} 28 \mathrm{~B}$ & 109.5 \\
\hline $\mathrm{O} 5-\mathrm{C} 5-\mathrm{H} 5 \mathrm{~A}$ & 109.5 & $\mathrm{H} 28 \mathrm{~A}-\mathrm{C} 28-\mathrm{H} 28 \mathrm{~B}$ & 109.5 \\
\hline $\mathrm{C} 4-\mathrm{C} 5-\mathrm{H} 5 \mathrm{~A}$ & 109.5 & $\mathrm{O} 24-\mathrm{C} 28-\mathrm{H} 28 \mathrm{C}$ & 109.5 \\
\hline $\mathrm{C} 6-\mathrm{C} 5-\mathrm{H} 5 \mathrm{~A}$ & 109.5 & $\mathrm{H} 28 \mathrm{~A}-\mathrm{C} 28-\mathrm{H} 28 \mathrm{C}$ & 109.5 \\
\hline $\mathrm{O} 6-\mathrm{C} 6-\mathrm{C} 16$ & $109.3(3)$ & $\mathrm{H} 28 \mathrm{~B}-\mathrm{C} 28-\mathrm{H} 28 \mathrm{C}$ & 109.5 \\
\hline $\mathrm{O} 6-\mathrm{C} 6-\mathrm{C} 7$ & $107.0(3)$ & $\mathrm{C} 26-\mathrm{C} 29-\mathrm{H} 29 \mathrm{~A}$ & 109.5 \\
\hline $\mathrm{C} 16-\mathrm{C} 6-\mathrm{C} 7$ & $112.6(3)$ & $\mathrm{C} 26-\mathrm{C} 29-\mathrm{H} 29 \mathrm{~B}$ & 109.5 \\
\hline $\mathrm{O} 6-\mathrm{C} 6-\mathrm{C} 5$ & $109.6(3)$ & $\mathrm{H} 29 \mathrm{~A}-\mathrm{C} 29-\mathrm{H} 29 \mathrm{~B}$ & 109.5 \\
\hline $\mathrm{C} 16-\mathrm{C} 6-\mathrm{C} 5$ & $109.9(3)$ & $\mathrm{C} 26-\mathrm{C} 29-\mathrm{H} 29 \mathrm{C}$ & 109.5 \\
\hline $\mathrm{C} 7-\mathrm{C} 6-\mathrm{C} 5$ & $108.4(3)$ & $\mathrm{H} 29 \mathrm{~A}-\mathrm{C} 29-\mathrm{H} 29 \mathrm{C}$ & 109.5 \\
\hline $\mathrm{C} 8-\mathrm{C} 7-\mathrm{C} 6$ & $117.4(3)$ & $\mathrm{H} 29 \mathrm{~B}-\mathrm{C} 29-\mathrm{H} 29 \mathrm{C}$ & 109.5 \\
\hline $\mathrm{C} 8-\mathrm{C} 7-\mathrm{H} 7 \mathrm{~A}$ & 108.0 & $\mathrm{O} 5-\mathrm{C} 30-\mathrm{O} 30$ & $108.7(2)$ \\
\hline $\mathrm{C} 6-\mathrm{C} 7-\mathrm{H} 7 \mathrm{~A}$ & 108.0 & $\mathrm{O} 5-\mathrm{C} 30-\mathrm{C} 34$ & $105.3(2)$ \\
\hline $\mathrm{C} 8-\mathrm{C} 7-\mathrm{H} 7 \mathrm{~B}$ & 108.0 & $\mathrm{O} 30-\mathrm{C} 30-\mathrm{C} 34$ & $110.5(2)$ \\
\hline $\mathrm{C} 6-\mathrm{C} 7-\mathrm{H} 7 \mathrm{~B}$ & 108.0 & $\mathrm{O} 5-\mathrm{C} 30-\mathrm{H} 30 \mathrm{~A}$ & 110.7 \\
\hline $\mathrm{H} 7 \mathrm{~A}-\mathrm{C} 7-\mathrm{H} 7 \mathrm{~B}$ & 107.2 & $\mathrm{O} 30-\mathrm{C} 30-\mathrm{H} 30 \mathrm{~A}$ & 110.7 \\
\hline $\mathrm{C} 9-\mathrm{C} 8-\mathrm{C} 17$ & $111.7(3)$ & $\mathrm{C} 34-\mathrm{C} 30-\mathrm{H} 30 \mathrm{~A}$ & 110.7 \\
\hline $\mathrm{C} 9-\mathrm{C} 8-\mathrm{C} 7$ & $108.2(3)$ & $\mathrm{O} 30-\mathrm{C} 31-\mathrm{C} 35$ & $107.1(3)$ \\
\hline $\mathrm{C} 17-\mathrm{C} 8-\mathrm{C} 7$ & $112.9(3)$ & $\mathrm{O} 30-\mathrm{C} 31-\mathrm{C} 32$ & $109.6(3)$ \\
\hline $\mathrm{C} 9-\mathrm{C} 8-\mathrm{H} 8 \mathrm{~A}$ & 107.9 & $\mathrm{C} 35-\mathrm{C} 31-\mathrm{C} 32$ & $112.8(3)$ \\
\hline $\mathrm{C} 17-\mathrm{C} 8-\mathrm{H} 8 \mathrm{~A}$ & 107.9 & $\mathrm{O} 30-\mathrm{C} 31-\mathrm{H} 31 \mathrm{~A}$ & 109.1 \\
\hline $\mathrm{C} 7-\mathrm{C} 8-\mathrm{H} 8 \mathrm{~A}$ & 107.9 & $\mathrm{C} 35-\mathrm{C} 31-\mathrm{H} 31 \mathrm{~A}$ & 109.1 \\
\hline $\mathrm{O} 9-\mathrm{C} 9-\mathrm{C} 8$ & $120.9(4)$ & $\mathrm{C} 32-\mathrm{C} 31-\mathrm{H} 31 \mathrm{~A}$ & 109.1 \\
\hline $\mathrm{O} 9-\mathrm{C} 9-\mathrm{C} 10$ & $119.8(4)$ & $\mathrm{C} 31-\mathrm{C} 32-\mathrm{C} 33$ & $112.3(3)$ \\
\hline $\mathrm{C} 8-\mathrm{C} 9-\mathrm{C} 10$ & $119.3(3)$ & $\mathrm{C} 31-\mathrm{C} 32-\mathrm{H} 32 \mathrm{~A}$ & 109.2 \\
\hline $\mathrm{C} 9-\mathrm{C} 10-\mathrm{C} 11$ & $109.9(3)$ & $\mathrm{C} 33-\mathrm{C} 32-\mathrm{H} 32 \mathrm{~A}$ & 109.2 \\
\hline $\mathrm{C} 9-\mathrm{C} 10-\mathrm{C} 18$ & $109.7(3)$ & $\mathrm{C} 31-\mathrm{C} 32-\mathrm{H} 32 \mathrm{~B}$ & 109.2 \\
\hline $\mathrm{C} 11-\mathrm{C} 10-\mathrm{C} 18$ & $115.0(3)$ & $\mathrm{C} 33-\mathrm{C} 32-\mathrm{H} 32 \mathrm{~B}$ & 109.2 \\
\hline $\mathrm{C} 9-\mathrm{C} 10-\mathrm{H} 10 \mathrm{~A}$ & 107.3 & $\mathrm{H} 32 \mathrm{~A}-\mathrm{C} 32-\mathrm{H} 32 \mathrm{~B}$ & 107.9 \\
\hline $\mathrm{C} 11-\mathrm{C} 10-\mathrm{H} 10 \mathrm{~A}$ & 107.3 & N33-C33-C34 & $110.3(3)$ \\
\hline
\end{tabular}




\begin{tabular}{|c|c|}
\hline $\mathrm{C} 18-\mathrm{C} 10-\mathrm{H} 10 \mathrm{~A}$ & 107.3 \\
\hline $\mathrm{O} 11-\mathrm{C} 11-\mathrm{C} 12$ & $109.3(3)$ \\
\hline $\mathrm{O} 11-\mathrm{C} 11-\mathrm{C} 10$ & $108.5(3)$ \\
\hline $\mathrm{C} 12-\mathrm{C} 11-\mathrm{C} 10$ & $118.2(3)$ \\
\hline $\mathrm{O} 11-\mathrm{C} 11-\mathrm{H} 11 \mathrm{~A}$ & 106.8 \\
\hline $\mathrm{C} 12-\mathrm{C} 11-\mathrm{H} 11 \mathrm{~A}$ & 106.8 \\
\hline $\mathrm{C} 10-\mathrm{C} 11-\mathrm{H} 11 \mathrm{~A}$ & 106.8 \\
\hline $\mathrm{O} 12-\mathrm{C} 12-\mathrm{C} 19$ & $112.7(3)$ \\
\hline $\mathrm{O} 12-\mathrm{C} 12-\mathrm{C} 11$ & $105.6(3)$ \\
\hline $\mathrm{C} 19-\mathrm{C} 12-\mathrm{C} 11$ & $113.9(3)$ \\
\hline $\mathrm{O} 12-\mathrm{C} 12-\mathrm{C} 13$ & $104.8(3)$ \\
\hline $\mathrm{C} 19-\mathrm{C} 12-\mathrm{C} 13$ & $110.9(3)$ \\
\hline $\mathrm{C} 11-\mathrm{C} 12-\mathrm{C} 13$ & 108.5 \\
\hline $\mathrm{O} 13-\mathrm{C} 13-\mathrm{C} 20$ & $106.2(3)$ \\
\hline $\mathrm{O} 13-\mathrm{C} 13-\mathrm{C} 12$ & $108.1(3)$ \\
\hline $\mathrm{C} 20-\mathrm{C} 13-\mathrm{C} 12$ & $115.5(3)$ \\
\hline $\mathrm{O} 13-\mathrm{C} 13-\mathrm{H} 13 \mathrm{~A}$ & 108.9 \\
\hline $\mathrm{C} 20-\mathrm{C} 13-\mathrm{H} 13 \mathrm{~A}$ & 108.9 \\
\hline $\mathrm{C} 12-\mathrm{C} 13-\mathrm{H} 13 \mathrm{~A}$ & 108.9 \\
\hline $\mathrm{C} 2-\mathrm{C} 14-\mathrm{H} 14 \mathrm{~A}$ & 109.5 \\
\hline $\mathrm{C} 2-\mathrm{C} 14-\mathrm{H} 14 \mathrm{~B}$ & 109.5 \\
\hline $\mathrm{H} 14 \mathrm{~A}-\mathrm{C} 14-\mathrm{H} 14 \mathrm{~B}$ & 109.5 \\
\hline $\mathrm{C} 2-\mathrm{C} 14-\mathrm{H} 14 \mathrm{C}$ & 109.5 \\
\hline $\mathrm{H} 14 \mathrm{~A}-\mathrm{C} 14-\mathrm{H} 14 \mathrm{C}$ & 109.5 \\
\hline $\mathrm{H} 14 \mathrm{~B}-\mathrm{C} 14-\mathrm{H} 14 \mathrm{C}$ & 109.5 \\
\hline $\mathrm{C} 4-\mathrm{C} 15-\mathrm{H} 15 \mathrm{~A}$ & 109.5 \\
\hline C4-C15-H15B & 109.5 \\
\hline $\mathrm{H} 15 \mathrm{~A}-\mathrm{C} 15-\mathrm{H} 15 \mathrm{~B}$ & 109.5 \\
\hline $\mathrm{C} 4-\mathrm{C} 15-\mathrm{H} 15 \mathrm{C}$ & 109.5 \\
\hline $\mathrm{H} 15 \mathrm{~A}-\mathrm{C} 15-\mathrm{H} 15 \mathrm{C}$ & 109.5 \\
\hline $\mathrm{H} 15 \mathrm{~B}-\mathrm{C} 15-\mathrm{H} 15 \mathrm{C}$ & 109.5 \\
\hline $\mathrm{C} 6-\mathrm{C} 16-\mathrm{H} 16 \mathrm{~A}$ & 109.5 \\
\hline $\mathrm{C} 6-\mathrm{C} 16-\mathrm{H} 16 \mathrm{~B}$ & 109.5 \\
\hline $\mathrm{H} 16 \mathrm{~A}-\mathrm{C} 16-\mathrm{H} 16 \mathrm{~B}$ & 109.5 \\
\hline $\mathrm{C} 6-\mathrm{C} 16-\mathrm{H} 16 \mathrm{C}$ & 109.5 \\
\hline $\mathrm{H} 16 \mathrm{~A}-\mathrm{C} 16-\mathrm{H} 16 \mathrm{C}$ & 109.5 \\
\hline $\mathrm{H} 16 \mathrm{~B}-\mathrm{C} 16-\mathrm{H} 16 \mathrm{C}$ & 109.5 \\
\hline $\mathrm{C} 8-\mathrm{C} 17-\mathrm{H} 17 \mathrm{~A}$ & 109.5 \\
\hline $\mathrm{C} 8-\mathrm{C} 17-\mathrm{H} 17 \mathrm{~B}$ & 109.5 \\
\hline $\mathrm{H} 17 \mathrm{~A}-\mathrm{C} 17-\mathrm{H} 17 \mathrm{~B}$ & 109.5 \\
\hline $\mathrm{C} 8-\mathrm{C} 17-\mathrm{H} 17 \mathrm{C}$ & 109.5 \\
\hline $\mathrm{H} 17 \mathrm{~A}-\mathrm{C} 17-\mathrm{H} 17 \mathrm{C}$ & 109.5 \\
\hline $\mathrm{H} 17 \mathrm{~B}-\mathrm{C} 17-\mathrm{H} 17 \mathrm{C}$ & 109.5 \\
\hline $\mathrm{C} 10-\mathrm{C} 18-\mathrm{H} 18 \mathrm{~A}$ & 109.5 \\
\hline $\mathrm{C} 10-\mathrm{C} 18-\mathrm{H} 18 \mathrm{~B}$ & 109.5 \\
\hline $\mathrm{H} 18 \mathrm{~A}-\mathrm{C} 18-\mathrm{H} 18 \mathrm{~B}$ & 109.5 \\
\hline $\mathrm{C} 10-\mathrm{C} 18-\mathrm{H} 18 \mathrm{C}$ & 109.5 \\
\hline $\mathrm{H} 18 \mathrm{~A}-\mathrm{C} 18-\mathrm{H} 18 \mathrm{C}$ & 109.5 \\
\hline
\end{tabular}

\begin{tabular}{|c|c|}
\hline $\mathrm{N} 33-\mathrm{C} 33-\mathrm{C} 32$ & $115.9(3)$ \\
\hline $\mathrm{C} 34-\mathrm{C} 33-\mathrm{C} 32$ & $110.1(3)$ \\
\hline N33-C33-H33A & 106.7 \\
\hline C $34-\mathrm{C} 33-\mathrm{H} 33 \mathrm{~A}$ & 106.7 \\
\hline $\mathrm{C} 32-\mathrm{C} 33-\mathrm{H} 33 \mathrm{~A}$ & 106.7 \\
\hline $\mathrm{O} 34-\mathrm{C} 34-\mathrm{C} 33$ & $109.0(3)$ \\
\hline $\mathrm{O} 34-\mathrm{C} 34-\mathrm{C} 30$ & $111.4(3)$ \\
\hline $\mathrm{C} 33-\mathrm{C} 34-\mathrm{C} 30$ & $111.5(3)$ \\
\hline $\mathrm{O} 34-\mathrm{C} 34-\mathrm{H} 34 \mathrm{~A}$ & 108.3 \\
\hline $\mathrm{C} 33-\mathrm{C} 34-\mathrm{H} 34 \mathrm{~A}$ & 108.3 \\
\hline $\mathrm{C} 30-\mathrm{C} 34-\mathrm{H} 34 \mathrm{~A}$ & 108.3 \\
\hline $\mathrm{C} 31-\mathrm{C} 35-\mathrm{H} 35 \mathrm{~A}$ & 109.5 \\
\hline $\mathrm{C} 31-\mathrm{C} 35-\mathrm{H} 35 \mathrm{~B}$ & 109.5 \\
\hline $\mathrm{H} 35 \mathrm{~A}-\mathrm{C} 35-\mathrm{H} 35 \mathrm{~B}$ & 109.5 \\
\hline $\mathrm{C} 31-\mathrm{C} 35-\mathrm{H} 35 \mathrm{C}$ & 109.5 \\
\hline $\mathrm{H} 35 \mathrm{~A}-\mathrm{C} 35-\mathrm{H} 35 \mathrm{C}$ & 109.5 \\
\hline $\mathrm{H} 35 \mathrm{~B}-\mathrm{C} 35-\mathrm{H} 35 \mathrm{C}$ & 109.5 \\
\hline N33-C36-H36A & 109.5 \\
\hline N33-C36- H36B & 109.5 \\
\hline $\mathrm{H} 36 \mathrm{~A}-\mathrm{C} 36-\mathrm{H} 36 \mathrm{~B}$ & 109.5 \\
\hline $\mathrm{N} 33-\mathrm{C} 36-\mathrm{H} 36 \mathrm{C}$ & 109.5 \\
\hline $\mathrm{H} 36 \mathrm{~A}-\mathrm{C} 36-\mathrm{H} 36 \mathrm{C}$ & 109.5 \\
\hline $\mathrm{H} 36 \mathrm{~B}-\mathrm{C} 36-\mathrm{H} 36 \mathrm{C}$ & 109.5 \\
\hline N33-C37-H37A & 109.5 \\
\hline N33-C37-H37B & 109.5 \\
\hline $\mathrm{H} 37 \mathrm{~A}-\mathrm{C} 37-\mathrm{H} 37 \mathrm{~B}$ & 109.5 \\
\hline N33-C37-H37C & 109.5 \\
\hline $\mathrm{H} 37 \mathrm{~A}-\mathrm{C} 37-\mathrm{H} 37 \mathrm{C}$ & 109.5 \\
\hline $\mathrm{H} 37 \mathrm{~B}-\mathrm{C} 37-\mathrm{H} 37 \mathrm{C}$ & 109.5 \\
\hline $\mathrm{O} 14-\mathrm{S} 1-\mathrm{C} 38$ & $106.4(3)$ \\
\hline $\mathrm{O} 14-\mathrm{S} 1-\mathrm{C} 39$ & $107.0(2)$ \\
\hline $\mathrm{C} 38-\mathrm{S} 1-\mathrm{C} 39$ & $96.9(3)$ \\
\hline $\mathrm{S} 1-\mathrm{C} 38-\mathrm{H} 38 \mathrm{~A}$ & 109.5 \\
\hline $\mathrm{S} 1-\mathrm{C} 38-\mathrm{H} 38 \mathrm{~B}$ & 109.5 \\
\hline $\mathrm{H} 38 \mathrm{~A}-\mathrm{C} 38-\mathrm{H} 38 \mathrm{~B}$ & 109.5 \\
\hline $\mathrm{S} 1-\mathrm{C} 38-\mathrm{H} 38 \mathrm{C}$ & 109.5 \\
\hline $\mathrm{H} 38 \mathrm{~A}-\mathrm{C} 38-\mathrm{H} 38 \mathrm{C}$ & 109.5 \\
\hline $\mathrm{H} 38 \mathrm{~B}-\mathrm{C} 38-\mathrm{H} 38 \mathrm{C}$ & 109.5 \\
\hline $\mathrm{S} 1-\mathrm{C} 39-\mathrm{H} 39 \mathrm{~A}$ & 109.5 \\
\hline $\mathrm{S} 1-\mathrm{C} 39-\mathrm{H} 39 \mathrm{~B}$ & 109.5 \\
\hline $\mathrm{H} 39 \mathrm{~A}-\mathrm{C} 39-\mathrm{H} 39 \mathrm{~B}$ & 109.5 \\
\hline $\mathrm{S} 1-\mathrm{C} 39-\mathrm{H} 39 \mathrm{C}$ & 109.5 \\
\hline $\mathrm{H} 39 \mathrm{~A}-\mathrm{C} 39-\mathrm{H} 39 \mathrm{C}$ & 109.5 \\
\hline $\mathrm{H} 39 \mathrm{~B}-\mathrm{C} 39-\mathrm{H} 39 \mathrm{C}$ & 109.5 \\
\hline $\mathrm{O} 15-\mathrm{S} 2-\mathrm{C} 41$ & $105.7(5)$ \\
\hline $\mathrm{O} 15-\mathrm{S} 2-\mathrm{C} 40$ & $103.7(6)$ \\
\hline $\mathrm{C} 41-\mathrm{S} 2-\mathrm{C} 40$ & $99.7(9)$ \\
\hline $\mathrm{S} 2-\mathrm{C} 40-\mathrm{H} 40 \mathrm{D}$ & 109.5 \\
\hline
\end{tabular}


H18B-C18-H18C

C12-C19-H19A

C12-C19-H19B

H19A-C19-H19B

C12-C19-H19C

$\mathrm{H} 19 \mathrm{~A}-\mathrm{C} 19-\mathrm{H} 19 \mathrm{C}$

$\mathrm{H} 19 \mathrm{~B}-\mathrm{C} 19-\mathrm{H} 19 \mathrm{C}$

$\mathrm{C} 21-\mathrm{C} 20-\mathrm{C} 13$

$\mathrm{C} 21-\mathrm{C} 20-\mathrm{H} 20 \mathrm{~A}$

$\mathrm{C} 13-\mathrm{C} 20-\mathrm{H} 20 \mathrm{~A}$

$\mathrm{C} 21-\mathrm{C} 20-\mathrm{H} 20 \mathrm{~B}$

$\mathrm{C} 13-\mathrm{C} 20-\mathrm{H} 20 \mathrm{~B}$

$\mathrm{H} 20 \mathrm{~A}-\mathrm{C} 20-\mathrm{H} 20 \mathrm{~B}$

$\mathrm{C} 20-\mathrm{C} 21-\mathrm{H} 21 \mathrm{~A}$

$\mathrm{C} 20-\mathrm{C} 21-\mathrm{H} 21 \mathrm{~B}$

$\mathrm{H} 21 \mathrm{~A}-\mathrm{C} 21-\mathrm{H} 21 \mathrm{~B}$

$\mathrm{C} 20-\mathrm{C} 21-\mathrm{H} 21 \mathrm{C}$

$\mathrm{H} 21 \mathrm{~A}-\mathrm{C} 21-\mathrm{H} 21 \mathrm{C}$

$\mathrm{C} 13-\mathrm{O} 13-\mathrm{C} 1-\mathrm{O} 1$

$\mathrm{C} 13-\mathrm{O} 13-\mathrm{C} 1-\mathrm{C} 2$

$\mathrm{O} 1-\mathrm{C} 1-\mathrm{C} 2-\mathrm{C} 14$

$\mathrm{O} 13-\mathrm{C} 1-\mathrm{C} 2-\mathrm{C} 14$

$\mathrm{O} 1-\mathrm{C} 1-\mathrm{C} 2-\mathrm{C} 3$

$\mathrm{O} 13-\mathrm{C} 1-\mathrm{C} 2-\mathrm{C} 3$

$\mathrm{C} 22-\mathrm{O} 3-\mathrm{C} 3-\mathrm{C} 2$

$\mathrm{C} 22-\mathrm{O} 3-\mathrm{C} 3-\mathrm{C} 4$

$\mathrm{C} 14-\mathrm{C} 2-\mathrm{C} 3-\mathrm{O} 3$

$\mathrm{C} 1-\mathrm{C} 2-\mathrm{C} 3-\mathrm{O} 3$

$\mathrm{C} 14-\mathrm{C} 2-\mathrm{C} 3-\mathrm{C} 4$

$\mathrm{C} 1-\mathrm{C} 2-\mathrm{C} 3-\mathrm{C} 4$

$\mathrm{O} 3-\mathrm{C} 3-\mathrm{C} 4-\mathrm{C} 15$

$\mathrm{C} 2-\mathrm{C} 3-\mathrm{C} 4-\mathrm{C} 15$

$\mathrm{O} 3-\mathrm{C} 3-\mathrm{C} 4-\mathrm{C} 5$

$\mathrm{C} 2-\mathrm{C} 3-\mathrm{C} 4-\mathrm{C} 5$

$\mathrm{C} 30-\mathrm{O} 5-\mathrm{C} 5-\mathrm{C} 4$

$\mathrm{C} 30-\mathrm{O} 5-\mathrm{C} 5-\mathrm{C} 6$

$\mathrm{C} 3-\mathrm{C} 4-\mathrm{C} 5-\mathrm{O} 5$

$\mathrm{C} 15-\mathrm{C} 4-\mathrm{C} 5-\mathrm{O} 5$

$\mathrm{C} 3-\mathrm{C} 4-\mathrm{C} 5-\mathrm{C} 6$

$\mathrm{C} 15-\mathrm{C} 4-\mathrm{C} 5-\mathrm{C} 6$

$\mathrm{O} 5-\mathrm{C} 5-\mathrm{C} 6-\mathrm{O} 6$

$\mathrm{C} 4-\mathrm{C} 5-\mathrm{C} 6-\mathrm{O} 6$

$\mathrm{O} 5-\mathrm{C} 5-\mathrm{C} 6-\mathrm{C} 16$

$\mathrm{C} 4-\mathrm{C} 5-\mathrm{C} 6-\mathrm{C} 16$

$\mathrm{O} 5-\mathrm{C} 5-\mathrm{C} 6-\mathrm{C} 7$

$\mathrm{C} 4-\mathrm{C} 5-\mathrm{C} 6-\mathrm{C} 7$

$\mathrm{O} 6-\mathrm{C} 6-\mathrm{C} 7-\mathrm{C} 8$
109.5

109.5

109.5

109.5

109.5

109.5

109.5

$112.9(4)$

109.0

109.0

109.0

109.0

107.8

109.5

109.5

109.5

109.5

109.5

$-7.9(5)$

$170.3(3)$

49.6 (4)

$-128.6(3)$

-74.1 (4)

107.8 (3)

$-103.4(3)$

$133.6(3)$

$61.1(3)$

$-178.0(2)$

$-178.7(3)$

$-57.8(3)$

$49.2(3)$

$-70.8(3)$

$-78.2(3)$

$161.7(2)$

$-105.4(3)$

$132.3(3)$

$157.8(2)$

$31.6(4)$

$-86.4(3)$

$147.4(3)$

$163.7(2)$

$43.3(3)$

$-76.2(3)$

$163.4(3)$

$47.3(3)$

$-73.1(3)$

27.4 (4)
$\mathrm{S} 2-\mathrm{C} 40-\mathrm{H} 40 \mathrm{E}$

$\mathrm{H} 40 \mathrm{D}-\mathrm{C} 40-\mathrm{H} 40 \mathrm{E}$

$\mathrm{S} 2-\mathrm{C} 40-\mathrm{H} 40 \mathrm{~F}$

$\mathrm{H} 40 \mathrm{D}-\mathrm{C} 40-\mathrm{H} 40 \mathrm{~F}$

$\mathrm{H} 40 \mathrm{E}-\mathrm{C} 40-\mathrm{H} 40 \mathrm{~F}$

$\mathrm{S} 2-\mathrm{C} 41-\mathrm{H} 41 \mathrm{~A}$

$\mathrm{S} 2-\mathrm{C} 41-\mathrm{H} 41 \mathrm{~B}$

$\mathrm{H} 41 \mathrm{~A}-\mathrm{C} 41-\mathrm{H} 41 \mathrm{~B}$

$\mathrm{S} 2-\mathrm{C} 41-\mathrm{H} 41 \mathrm{C}$

$\mathrm{H} 41 \mathrm{~A}-\mathrm{C} 41-\mathrm{H} 41 \mathrm{C}$

$\mathrm{H} 41 \mathrm{~B}-\mathrm{C} 41-\mathrm{H} 41 \mathrm{C}$

$\mathrm{H} 40 \mathrm{~A}-\mathrm{C} 40^{\prime}-\mathrm{H} 40 \mathrm{~B}$

$\mathrm{H} 40 \mathrm{~A}-\mathrm{C} 40^{\prime}-\mathrm{H} 40 \mathrm{C}$

$\mathrm{H} 40 \mathrm{~B}-\mathrm{C} 40^{\prime}-\mathrm{H} 40 \mathrm{C}$

$\mathrm{H} 41 \mathrm{D}-\mathrm{C} 41^{\prime}-\mathrm{H} 41 \mathrm{E}$

H41D-C41'-H41F

$\mathrm{H} 41 \mathrm{E}-\mathrm{C} 41^{\prime}-\mathrm{H} 41 \mathrm{~F}$

$\mathrm{C} 19-\mathrm{C} 12-\mathrm{C} 13-\mathrm{O} 13$

$\mathrm{C} 11-\mathrm{C} 12-\mathrm{C} 13-\mathrm{O} 13$

$\mathrm{O} 12-\mathrm{C} 12-\mathrm{C} 13-\mathrm{C} 20$

$\mathrm{C} 19-\mathrm{C} 12-\mathrm{C} 13-\mathrm{C} 20$

$\mathrm{C} 11-\mathrm{C} 12-\mathrm{C} 13-\mathrm{C} 20$

$\mathrm{O} 13-\mathrm{C} 13-\mathrm{C} 20-\mathrm{C} 21$

$\mathrm{C} 12-\mathrm{C} 13-\mathrm{C} 20-\mathrm{C} 21$

$\mathrm{C} 3-\mathrm{O} 3-\mathrm{C} 22-\mathrm{O} 26$

$\mathrm{C} 3-\mathrm{O} 3-\mathrm{C} 22-\mathrm{C} 23$

$\mathrm{C} 26-\mathrm{O} 26-\mathrm{C} 22-\mathrm{O} 3$

$\mathrm{C} 26-\mathrm{O} 26-\mathrm{C} 22-\mathrm{C} 23$

$\mathrm{O} 3-\mathrm{C} 22-\mathrm{C} 23-\mathrm{C} 24$

$\mathrm{O} 26-\mathrm{C} 22-\mathrm{C} 23-\mathrm{C} 24$

$\mathrm{C} 28-\mathrm{O} 24-\mathrm{C} 24-\mathrm{C} 25$

$\mathrm{C} 28-\mathrm{O} 24-\mathrm{C} 24-\mathrm{C} 23$

$\mathrm{C} 28-\mathrm{O} 24-\mathrm{C} 24-\mathrm{C} 27$

$\mathrm{C} 22-\mathrm{C} 23-\mathrm{C} 24-\mathrm{O} 24$

$\mathrm{C} 22-\mathrm{C} 23-\mathrm{C} 24-\mathrm{C} 25$

$\mathrm{C} 22-\mathrm{C} 23-\mathrm{C} 24-\mathrm{C} 27$

$\mathrm{O} 24-\mathrm{C} 24-\mathrm{C} 25-\mathrm{O} 25$

$\mathrm{C} 23-\mathrm{C} 24-\mathrm{C} 25-\mathrm{O} 25$

$\mathrm{C} 27-\mathrm{C} 24-\mathrm{C} 25-\mathrm{O} 25$

$\mathrm{O} 24-\mathrm{C} 24-\mathrm{C} 25-\mathrm{C} 26$

$\mathrm{C} 23-\mathrm{C} 24-\mathrm{C} 25-\mathrm{C} 26$

$\mathrm{C} 27-\mathrm{C} 24-\mathrm{C} 25-\mathrm{C} 26$

$\mathrm{C} 22-\mathrm{O} 26-\mathrm{C} 26-\mathrm{C} 25$

$\mathrm{C} 22-\mathrm{O} 26-\mathrm{C} 26-\mathrm{C} 29$

$\mathrm{O} 25-\mathrm{C} 25-\mathrm{C} 26-\mathrm{O} 26$

$\mathrm{C} 24-\mathrm{C} 25-\mathrm{C} 26-\mathrm{O} 26$
109.5

109.5

109.5

109.5

109.5

109.5

109.5

109.5

109.5

109.5

109.5

109.5

109.5

109.5

109.5

109.5

109.5

$50.4(4)$

-75.4 (3)

53.5 (4)

-68.4 (4)

$165.8(3)$

70.1 (4)

$-170.1(3)$

-83.3 (3)

151.0 (3)

-75.0 (3)

49.9 (4)

79.8 (4)

-45.6 (4)

$-171.9(3)$

-55.2 (4)

68.7 (4)

-66.5 (4)

48.4 (4)

169.1 (3)

-61.2 (3)

178.1 (3)

58.0 (4)

65.4 (3)

-55.2 (4)

$-175.3(3)$

-58.2 (3)

-180.0 (3)

-171.7 (3)

60.9 (4) 


$\begin{array}{ll}\mathrm{C} 16-\mathrm{C} 6-\mathrm{C} 7-\mathrm{C} 8 & -92.7(4) \\ \mathrm{C} 5-\mathrm{C} 6-\mathrm{C} 7-\mathrm{C} 8 & 145.5(3) \\ \mathrm{C} 6-\mathrm{C} 7-\mathrm{C} 8-\mathrm{C} 9 & -165.1(3) \\ \mathrm{C} 6-\mathrm{C} 7-\mathrm{C} 8-\mathrm{C} 17 & 70.7(5) \\ \mathrm{C} 17-\mathrm{C} 8-\mathrm{C} 9-\mathrm{O} 9 & 9.0(5) \\ \mathrm{C} 7-\mathrm{C} 8-\mathrm{C} 9-\mathrm{O} 9 & -115.9(4) \\ \mathrm{C} 17-\mathrm{C} 8-\mathrm{C} 9-\mathrm{C} 10 & -171.5(3) \\ \mathrm{C} 7-\mathrm{C} 8-\mathrm{C} 9-\mathrm{C} 10 & 63.6(4) \\ \mathrm{O} 9-\mathrm{C} 9-\mathrm{C} 10-\mathrm{C} 11 & -134.4(4) \\ \mathrm{C} 8-\mathrm{C} 9-\mathrm{C} 10-\mathrm{C} 11 & 46.0(4) \\ \mathrm{O} 9-\mathrm{C} 9-\mathrm{C} 10-\mathrm{C} 18 & -6.9(5) \\ \mathrm{C} 8-\mathrm{C} 9-\mathrm{C} 10-\mathrm{C} 18 & 173.5(3) \\ \mathrm{C} 9-\mathrm{C} 10-\mathrm{C} 11-\mathrm{O} 11 & 53.6(4) \\ \mathrm{C} 18-\mathrm{C} 10-\mathrm{C} 11-\mathrm{O} 11 & -70.9(4) \\ \mathrm{C} 9-\mathrm{C} 10-\mathrm{C} 11-\mathrm{C} 12 & 178.6(3) \\ \mathrm{C} 18-\mathrm{C} 10-\mathrm{C} 11-\mathrm{C} 12 & 54.2(4) \\ \mathrm{O} 11-\mathrm{C} 11-\mathrm{C} 12-\mathrm{O} 12 & 40.4(4) \\ \mathrm{C} 10-\mathrm{C} 11-\mathrm{C} 12-\mathrm{O} 12 & -84.3(4) \\ \mathrm{O} 11-\mathrm{C} 11-\mathrm{C} 12-\mathrm{C} 19 & 164.5(3) \\ \mathrm{C} 10-\mathrm{C} 11-\mathrm{C} 12-\mathrm{C} 19 & 39.9(4) \\ \mathrm{O} 11-\mathrm{C} 11-\mathrm{C} 12-\mathrm{C} 13 & -71.4(3) \\ \mathrm{C} 10-\mathrm{C} 11-\mathrm{C} 12-\mathrm{C} 13 & 163.9(3) \\ \mathrm{C} 1-\mathrm{O} 13-\mathrm{C} 13-\mathrm{C} 20 & -117.1(3) \\ \mathrm{C} 1-\mathrm{O} 13-\mathrm{C} 13-\mathrm{C} 12 & 118.3(3) \\ \mathrm{O} 12-\mathrm{C} 12-\mathrm{C} 13-\mathrm{O} 13 & 172.3(3) \\ \end{array}$

$\begin{array}{ll}\mathrm{O} 25-\mathrm{C} 25-\mathrm{C} 26-\mathrm{C} 29 & -53.9(4) \\ \mathrm{C} 24-\mathrm{C} 25-\mathrm{C} 26-\mathrm{C} 29 & 178.8(3) \\ \mathrm{C} 5-\mathrm{O} 5-\mathrm{C} 30-\mathrm{O} 30 & -74.8(3) \\ \mathrm{C} 5-\mathrm{O} 5-\mathrm{C} 30-\mathrm{C} 34 & 166.8(2) \\ \mathrm{C} 31-\mathrm{O} 30-\mathrm{C} 30-\mathrm{O} 5 & -176.7(3) \\ \mathrm{C} 31-\mathrm{O} 30-\mathrm{C} 30-\mathrm{C} 34 & -61.7(3) \\ \mathrm{C} 30-\mathrm{O} 30-\mathrm{C} 31-\mathrm{C} 35 & -176.0(3) \\ \mathrm{C} 30-\mathrm{O} 30-\mathrm{C} 31-\mathrm{C} 32 & 61.3(4) \\ \mathrm{O} 30-\mathrm{C} 31-\mathrm{C} 32-\mathrm{C} 33 & -55.0(4) \\ \mathrm{C} 35-\mathrm{C} 31-\mathrm{C} 32-\mathrm{C} 33 & -174.2(3) \\ \mathrm{C} 37-\mathrm{N} 33-\mathrm{C} 33-\mathrm{C} 34 & 58.7(4) \\ \mathrm{C} 36-\mathrm{N} 33-\mathrm{C} 33-\mathrm{C} 34 & -175.7(3) \\ \mathrm{C} 37-\mathrm{N} 33-\mathrm{C} 33-\mathrm{C} 32 & -67.3(4) \\ \mathrm{C} 36-\mathrm{N} 33-\mathrm{C} 33-\mathrm{C} 32 & 58.3(4) \\ \mathrm{C} 31-\mathrm{C} 32-\mathrm{C} 33-\mathrm{N} 33 & 176.4(3) \\ \mathrm{C} 31-\mathrm{C} 32-\mathrm{C} 33-\mathrm{C} 34 & 50.4(4) \\ \mathrm{N} 33-\mathrm{C} 33-\mathrm{C} 34-\mathrm{O} 34 & 57.6(3) \\ \mathrm{C} 32-\mathrm{C} 33-\mathrm{C} 34-\mathrm{O} 34 & -173.3(3) \\ \mathrm{N} 33-\mathrm{C} 33-\mathrm{C} 34-\mathrm{C} 30 & -179.0(3) \\ \mathrm{C} 32-\mathrm{C} 33-\mathrm{C} 34-\mathrm{C} 30 & -49.9(4) \\ \mathrm{O} 5-\mathrm{C} 30-\mathrm{C} 34-\mathrm{O} 34 & -65.0(3) \\ \mathrm{O} 30-\mathrm{C} 30-\mathrm{C} 34-\mathrm{O} 34 & 177.9(2) \\ \mathrm{O} 5-\mathrm{C} 30-\mathrm{C} 34-\mathrm{C} 33 & 173.0(3) \\ \mathrm{O} 30-\mathrm{C} 30-\mathrm{C} 34-\mathrm{C} 33 & 55.8(3) \\ & \end{array}$

Hydrogen-bond geometry $\left(\AA,{ }^{\circ}\right)$

\begin{tabular}{lllll}
\hline$D-\mathrm{H}^{\prime} \cdots A$ & $D-\mathrm{H}$ & $\mathrm{H} \cdots A$ & $D \cdots A$ & $D-\mathrm{H}^{\cdots} A A$ \\
\hline $\mathrm{O} 6-\mathrm{H} 6 A \cdots \mathrm{O} 7 W^{\mathrm{i}}$ & 0.82 & 2.00 & $2.810(4)$ & 170 \\
$\mathrm{O} 7 W-\mathrm{H} 7 C \cdots \mathrm{O} 26^{\mathrm{ii}}$ & $0.85(1)$ & $2.07(2)$ & $2.888(4)$ & $163(4)$ \\
$\mathrm{O} 7 W-\mathrm{H} 7 D \cdots \mathrm{N} 33$ & $0.84(1)$ & $2.04(2)$ & $2.863(4)$ & $166(5)$ \\
$\mathrm{O} 11-\mathrm{H} 11 B \cdots \mathrm{O} 12$ & 0.82 & 2.07 & $2.575(4)$ & 120 \\
$\mathrm{O} 12-\mathrm{H} 12 A \cdots \mathrm{O} 15$ & 0.82 & 1.85 & $2.602(8)$ & 152 \\
$\mathrm{O} 12-\mathrm{H} 12 A \cdots \mathrm{O} 15^{\prime}$ & 0.82 & 2.04 & $2.667(7)$ & 134 \\
$\mathrm{O} 25-\mathrm{H} 25 B \cdots \mathrm{O} 11^{\mathrm{iii}}$ & 0.82 & 2.04 & $2.838(4)$ & 166 \\
$\mathrm{O} 34-\mathrm{H} 34 B \cdots \mathrm{O} 14$ & 0.82 & 1.94 & $2.747(4)$ & 170 \\
\hline
\end{tabular}

Symmetry codes: (i) $x+1, y, z$; (ii) $x-1, y, z$; (iii) $x, y, z+1$. 